\title{
On the explicit upper and lower bounds for the number of zeros of the Selberg class
}

\author{
Neea Palojärvi * \\ Department of Mathematics and Statistics, Åbo Akademi University, \\ Domkyrkotorget 1, 20500 Åbo, Finland, neea.palojarvi@abo.fi
}

\begin{abstract}
In this paper we prove explicit upper and lower bounds for the error term in the Riemann-von Mangoldt type formula for the number of zeros inside the critical strip. Furthermore, we also give examples of the bounds.
\end{abstract}

\section{Introduction}

The Selberg class $S$, defined by Selberg [13], consists of functions $\mathcal{L}(s)=$ $\sum_{n=1}^{\infty} \frac{a(n)}{n^{s}}$ which satisfy the following conditions:

1. Ramanujan hypothesis: For any $\epsilon>0$ we have $|a(n)| \ll_{\epsilon} n^{\epsilon}$.

2. Analytic continuation: There is an integer $k \geq 0$ such that $(s-1)^{k} \mathcal{L}(s)$ is an entire function of finite order.

3. Functional equation: There exists a positive integer $f$ and a real number $Q$ and for integer $j \in[1, f]$ there are positive real numbers $\lambda_{j}$ and complex numbers $\omega, \mu_{j}, d_{\mathcal{L}}=2 \sum_{j=1}^{f} \lambda_{j}, \lambda=\prod_{j=1}^{f} \lambda_{j}^{2 \lambda_{j}}$ where $|\omega|=1$ and $\Re\left(\mu_{j}\right) \geq 0$ which satisfy

$$
\Lambda_{\mathcal{L}}(s)=\omega \overline{\Lambda_{\mathcal{L}}(1-\bar{s})}
$$

where

$$
\Lambda_{\mathcal{L}}(s)=\mathcal{L}(s) Q^{s} \prod_{j=1}^{f} \Gamma\left(\lambda_{j} s+\mu_{j}\right) .
$$

${ }^{*}$ This work was supported by the Vilho, Yrjö and Kalle Väisälä Foundation of the Finnish Academy of Science and Letters. 
4. Euler product: We have

$$
\mathcal{L}(s)=\prod_{p} \mathcal{L}_{p}(s)
$$

where

$$
\mathcal{L}_{p}(s)=\exp \left(\sum_{l=1}^{\infty} \frac{b\left(p^{l}\right)}{p^{l s}}\right)
$$

with some coefficients $b\left(p^{l}\right)$ satisfying $b\left(p^{l}\right) \ll p^{l \theta}$ for some $\theta<\frac{1}{2}$.

We denote $s=\sigma+i t$ where $\sigma$ and $t$ are real numbers. We also choose the principal branch of the $\operatorname{logarithm} \log \mathcal{L}(s)$ on the real axis as $\sigma \rightarrow \infty$. For other points we use the analytic continuation of the logarithm.

The zeros $s=-\frac{l+\mu_{j}}{\lambda_{j}}$ where $l=0,1,2, \ldots$ and $j \in[1, f]$ of the function $\mathcal{L}(s)$ are called trivial zeros. We notice that for all trivial zeros it holds that $\sigma \leq 0$. Since we suppose that the function has an Euler product, we know that $\mathcal{L}(s)$ does not have zeros for which $\sigma>1$ and $a(1)=1$. The zeros which lie in the strip $0 \leq \sigma \leq 1$ are called non-trivial zeros. The function may have a trivial and a non-trivial zero at the same point. By [5] if $d_{\mathcal{L}}=0$ then $\mathcal{L}(s) \equiv 1$ and if $d_{\mathcal{L}}>0$ then $d_{\mathcal{L}} \geq 1$. Since the number of zeros for $\mathcal{L}(s) \equiv 1$, we concentrate to the cases $d_{\mathcal{L}} \geq 1$.

Riemann-von Mangoldt formula [22, 23] describes the number of the zeros of the Riemann zeta function inside the certain strip. According to this formula, the number of the zeros $\rho$ for which $0 \leq \Im(\rho) \leq T$ for a positive real number $T$ is

$$
\frac{T}{2 \pi} \log \frac{T}{2 \pi}-\frac{T}{2 \pi}+O(\log T) .
$$

A. Selberg [14] and A. Fujii [10] proved similar formulas for the zeros of the $L$-functions inside the critical strip for height $T$ and $[T, T+H]$ respectively. J. Steuding [17] and L. Smaljović [16] estimated the number of the zeros of the Selberg class functions. The number of the zeros $\rho$ of the function $\mathcal{L} \in S$, for which $0 \leq \Im(\rho) \leq T$ or $-T \leq \Im(\rho) \leq 0$, is

$$
\frac{d_{\mathcal{L}}}{2 \pi} T \log \frac{T}{e}+\frac{T}{2 \pi} \log \left(\lambda Q^{2}\right)+O(\log T) .
$$

In this paper we are interested in the non-trivial zeros for which $T_{0}<\Im(\rho) \leq$ $T$ or $-T \leq \Im(\rho)<T_{0}$ when $T>T_{0}$ is large enough. We prove that

$\left|\mathcal{N}_{\mathcal{L}}^{ \pm}\left(T_{0}, T\right)-\frac{d_{\mathcal{L}}}{2 \pi} T \log \frac{T}{e}-\frac{T}{2 \pi} \log \left(\lambda Q^{2}\right)\right|<c_{\mathcal{L}, 1} \log T+c_{\mathcal{L}, 2}\left(T_{0}\right)+\frac{c_{\mathcal{L}, 3}\left(T_{0}\right)}{T}$,

where $\mathcal{N}_{\mathcal{L}}^{ \pm}\left(T_{0}, T\right)$ denotes the number of the non-trivial zeros of the function $\mathcal{L} \in S$ for $T_{0}<\Im(\rho) \leq T$ or $-T \leq \Im(\rho)<T_{0}$ when $T>T_{0}$ is large enough. The terms $c_{\mathcal{L}, j}\left(T_{0}\right)$ are real numbers which depend on the function $\mathcal{L}$ and the 
number $T_{0}$ and the real number $c_{\mathcal{L}, 1}$ depends only on the function $\mathcal{L}$. This formula involves no unknown, undefined constants in the error term. This is proved in Theorem 5.2, In 1916 R. J. Backlund [1] proved similar formula for the Riemann zeta function. E. Carneiro and R. Finder [4, 8] proved an explicit bound for the function $S_{1}(t, \pi)=\frac{1}{\pi} \int_{\frac{1}{2}}^{\infty} \log |L(\sigma+i t, \pi)| d \sigma$, where $L(s, \pi)$ is in a subset of the $L$-functions, assuming generalized Riemann hypothesis. There are also explicit upper bounds for the number of the zeros for the Riemann zeta-function assuming Riemann hypothesis [2] and along the critical line [20] and $L$-functions [3. G. França and A. LeClair [9] proved an exact equation for the $n$th zero of the $L$-functions on the critical line. There are also explicit results for Dirichlet $L$-functions and Dedekind zeta-functions, see for example the papers from K. S. McCurley [12] and T. S. Trudgian [21].

In the Section 2 we prove lemmas which are used in the other sections. We also prove a formula which describes the sum of the real parts of the zeros inside certain strip. The sum depends on the four integrals of the function $\mathcal{L}(s)$. The main result follows from the estimates of the integrals. In the Sections 3 and 4 we estimate the integrals. In the Section 5 we combine the results of the Sections 2 , 3 and 4 and prove the main result. We follow the proofs of the article [17] and the chapters 6 and 7 of the book [18]. In the Section 6 we give examples of the main result.

\section{Preliminary results}

In this section we prove some preliminary results which are needed to prove the main theorem. The results are used in the Sections 3, 4 and 5.

\subsection{Basic theory of the function $\mathcal{L}(s)$}

To shorten our notation we define

$$
v=\sum_{j=1}^{f} \lambda_{j} \log \lambda_{j}, u=\sum_{j=1}^{f}\left(\bar{\mu}_{j}-\frac{1}{2}\right) \log \lambda_{j} \text { and } \mu=4 \sum_{j=1}^{f}\left(\frac{1}{2}-\mu_{j}\right) .
$$

By the Ramanujan hypothesis $(\epsilon=1)$ there is a constant $a_{1}$ such that for all $n$ we have $|a(n)| \leq a_{1} n$. Note that $a_{1} \geq 1$ since we have assumed that $a(n)=1$. Let $a$ be a real number for which $a>2$ and

$$
\sum_{n=2}^{\infty} \frac{a_{1}}{n^{a}}<\frac{1}{2}
$$

and let $b<-3$ be a negative real number which has the following property:

$$
\sum_{n=2}^{\infty} \frac{a_{1}}{n^{-b-1}}<1
$$


In the chapter 7.1 of the book [18] it is proved that:

Lemma 2.1. Let $T_{0}$ and $T>T_{0}$ be a positive real numbers. Let number $\rho$ denote the zero of the function $\mathcal{L} \in S$. Then

$$
\begin{aligned}
2 \pi \sum_{\substack{T_{0}<\Im(\rho) \leq T \\
\Re(\rho)>b}}(\Re(\rho)-b)= & \int_{T_{0}}^{T} \log |\mathcal{L}(b+i t)| d t-\int_{T_{0}}^{T} \log |\mathcal{L}(a+i t)| d t \\
& -\int_{b}^{a} \arg \mathcal{L}\left(\sigma+i T_{0}\right) d \sigma+\int_{b}^{a} \arg \mathcal{L}(\sigma+i T) d \sigma \\
:= & I_{1}\left(T_{0}, T, b\right)+I_{2}\left(T_{0}, T, a\right)-I_{3}\left(T_{0}, a, b\right)+I_{3}(T, a, b) .
\end{aligned}
$$

The goal is to estimate the integrals and get the main result by using these estimates. By the functional equation we have

$$
\mathcal{L}(s)=\Delta_{\mathcal{L}}(s) \overline{\mathcal{L}(1-\bar{s})}
$$

where

$$
\Delta_{\mathcal{L}}(s)=\omega Q^{1-2 s} \prod_{j=1}^{f} \frac{\Gamma\left(\lambda_{j}(1-s)+\bar{\mu}_{j}\right)}{\Gamma\left(\lambda_{j} s+\mu_{j}\right)} .
$$

We use this formula to estimate the term $\log \left|\Delta_{\mathcal{L}}(s)\right|$. We need this when we estimate the terms $I_{1}\left(T_{0}, T, b\right)-I_{1}\left(T_{0}, T, b+1\right), I_{3}\left(T_{0}, a, b\right)$ and $I_{3}(T, a, b)$. To do this we define that $B_{n}$ is $n$th Bernoulli number and we need the following lemma from T. J. Stieltjes [19, paragraph 9]:

Lemma 2.2. Let $z$ be a complex number such that $|\arg (z)|<\pi$. Then for $N=1,2, \ldots$

$$
\log \Gamma(z)=z \log z-z+\frac{1}{2} \log \frac{2 \pi}{z}+\sum_{n=1}^{N-1} \frac{B_{2 n}}{2 n(2 n-1) z^{2 n-1}}+W_{N}(z),
$$

where

$$
\left|W_{N}(z)\right| \leq \frac{B_{2 N}}{2 N(2 N-1)|z|^{2 N-1}} \sec ^{2 N}\left(\frac{\arg z}{2}\right)
$$

is a holomorphic function.

Let

$$
\begin{aligned}
V_{j}(s)= & \left(-\lambda_{j} s+\lambda_{j}+\bar{\mu}_{j}-\frac{1}{2}\right) \log \left(1+\frac{\lambda_{j}+\bar{\mu}_{j}}{-\lambda_{j} s}\right)-\left(\lambda_{j}+\bar{\mu}_{j}\right) \\
& -\left(\lambda_{j} s+\mu_{j}-\frac{1}{2}\right) \log \left(1+\frac{\mu_{j}}{\lambda_{j} s}\right)+\mu_{j}+W\left(-\lambda_{j} s\right)-W\left(\lambda_{j} s\right),
\end{aligned}
$$

where $|W(z)| \leq \frac{B_{2}}{2|z|} \sec ^{2}\left(\frac{\arg z}{2}\right)$ is a holomorphic function if $|\arg (z)|<\pi$. Let also $V(s)=\sum_{j=1}^{f} V_{j}(s)$. Using the previous lemma and simplifying expressions we get 
Lemma 2.3. Let $\left|\arg \left(\lambda_{j}(1-s)+\bar{\mu}_{j}\right)\right|<\pi,\left|\arg \left(\lambda_{j} s+\mu_{j}\right)\right|<\pi$ and $t>0$. Then

$$
\begin{aligned}
\log \left|\Delta_{\mathcal{L}}(s)\right|= & \left(\frac{1}{2}-\sigma\right)\left(d_{\mathcal{L}} \log t+\log \left(\lambda Q^{2}\right)\right)+d_{\mathcal{L}} \sigma \\
& +\Re\left(\log \left(1-\frac{\sigma i}{t}\right)\left(d_{\mathcal{L}}\left(\frac{1}{2}-s\right)+\frac{\Im(\mu) i}{2}\right)+V(s)\right)
\end{aligned}
$$

Proof. It is enough to estimate the product of the $\Gamma$-functions of the formula (11). First we do some basic calculation and then we use Lemma 2.2, After short computations we have

$$
\log \left(-\lambda_{j} s\right)=\log \left(\lambda_{j}\right)-\frac{\pi i}{2}+\log t+\log \left(1-\frac{\sigma i}{t}\right)
$$

and

$$
\log \left(\lambda_{j} s\right)=\log \left(\lambda_{j}\right)+\frac{\pi i}{2}+\log t+\log \left(1-\frac{\sigma i}{t}\right) .
$$

We apply Lemma 2.2 for $N=1$ and using the previous formulas, we get

$$
\begin{aligned}
\log & \left(\prod_{j=1}^{f} \frac{\Gamma\left(\lambda_{j}(1-s)+\bar{\mu}_{j}\right)}{\Gamma\left(\lambda_{j} s+\mu_{j}\right)}\right) \\
= & \sum_{j=1}^{f}\left(\left(\lambda_{j}-\lambda_{j} s+\bar{\mu}_{j}-\frac{1}{2}\right)\left(\log \left(\lambda_{j}\right)-\frac{\pi i}{2}+\log t+\log \left(1-\frac{\sigma i}{t}\right)\right)+\lambda_{j} s\right) \\
& -\sum_{j=1}^{f}\left(\left(\lambda_{j} s+\mu_{j}-\frac{1}{2}\right)\left(\log \left(\lambda_{j}\right)+\frac{\pi i}{2}+\log t+\log \left(1-\frac{\sigma i}{t}\right)\right)-\lambda_{j} s\right) \\
& +V(s) \\
= & \log t\left(d_{\mathcal{L}}\left(\frac{1}{2}-s\right)+\frac{\Im(\mu) i}{2}\right)+2 \Im(u) i+d_{\mathcal{L}} s+2 v\left(\frac{1}{2}-s\right) \\
& -\frac{\pi i}{4}\left(d_{\mathcal{L}}-\Re(\mu)\right)+\log \left(1-\frac{\sigma i}{t}\right)\left(d_{\mathcal{L}}\left(\frac{1}{2}-s\right)+\frac{\Im(\mu) i}{2}\right)+V(s) .
\end{aligned}
$$

The claim follows from the previous computations and the definition (1) of the function $\Delta_{\mathcal{L}}(s)$.

\subsection{The estimate of the function $V_{j}(s)$}

The formula of the function $\log \left|\Delta_{\mathcal{L}}(s)\right|$ in Lemma 2.3 contains the term $V(s)$. Thus we want also estimate the term $V(s)$. Since $V(s)=\sum_{j=1}^{f} V_{j}(s)$, it is sufficient to estimate the terms $V_{j}(s)$. Before doing this we prove a lemma. The estimate of the term $V_{j}(s)$ follows from this lemma. 
Lemma 2.4. If $t \geq \max _{j}\left\{\frac{2\left|\lambda_{j}+\bar{\mu}_{j}\right|}{\lambda_{j}}\right\}, t \geq \max _{j}\left\{\frac{2\left|\mu_{j}\right|}{\lambda_{j}}\right\}$ and $\sigma$ is a constant then for all $j$

$$
\begin{aligned}
& \left|W\left(-\lambda_{j} s\right)\right|+\left|W\left(\lambda_{j} s\right)\right| \\
& \quad<\frac{\left|B_{2}\right|}{2\left|\lambda_{j} t\right|}\left(2+\sec ^{2}\left(\frac{\arg \left(\lambda_{j}\left(-|\sigma|+\max _{j}\left\{\frac{2\left|\lambda_{j}+\bar{\mu}_{j}\right|}{\lambda_{j}}, \frac{2\left|\mu_{j}\right|}{\lambda_{j}}\right\} i\right)\right)}{2}\right)\right) .
\end{aligned}
$$

Proof. We assume that $\sigma<0$ since we can prove the case $\sigma \geq 0$ similarly. By the definition of the function $W(z)$ we have

$$
|W(z)| \leq \frac{B_{2}}{2|z|} \sec ^{2}\left(\frac{\arg z}{2}\right)
$$

if $|\arg (z)|<\pi$. Also, by the assumptions for the number $t$ we have $\left|\arg \left( \pm \lambda_{j} s\right)\right|<\pi$. The goal is to estimate the functions $\sec ^{2}\left(\frac{\arg \left(-\lambda_{j} s\right)}{2}\right)$ and $\sec ^{2}\left(\frac{\arg \left(\lambda_{j} s\right)}{2}\right)$. We do the estimate by using basic properties of the secant function.

First we estimate the arguments of the complex numbers $-\lambda_{j} s$ and $\lambda_{j} s$. Since $t \geq \max _{j}\left\{\frac{2\left|\lambda_{j}+\bar{\mu}_{j}\right|}{\lambda_{j}}\right\}, t \geq \max _{j}\left\{\frac{2\left|\mu_{j}\right|}{\lambda_{j}}\right\}$ and $\sigma<0$, the argument of the complex numbers $-\lambda_{j} s$ is in the interval $\left(-\frac{\pi}{2}, 0\right)$. Thus $\frac{\arg \left(-\lambda_{j} s\right)}{2} \in\left(-\frac{\pi}{4}, 0\right)$. Similarly we have

$$
\frac{\arg \left(\lambda_{j} s\right)}{2} \in\left(\frac{\pi}{4}, \frac{\arg \left(\lambda_{j}\left(\sigma+\max _{j}\left\{\frac{2\left|\lambda_{j}+\bar{\mu}_{j}\right|}{\lambda_{j}}, \frac{2\left|\mu_{j}\right|}{\lambda_{j}}\right\} i\right)\right)}{2}\right] \subset\left(\frac{\pi}{4}, \frac{\pi}{2}\right) .
$$

Next we use the estimates of the arguments. Because the function $\sec ^{2}(z)$ is an increasing function for $z \in\left[0, \frac{\pi}{2}\right)$ and an even function we have

$$
\sec ^{2}\left(\frac{\arg \left(-\lambda_{j} s\right)}{2}\right)<\sec ^{2}\left(-\frac{\pi}{4}\right)=2
$$

and

$$
\sec ^{2}\left(\frac{\arg \left(\lambda_{j} s\right)}{2}\right) \leq \sec ^{2}\left(\frac{\arg \left(\lambda_{j}\left(\sigma+\max _{j}\left\{\frac{2\left|\lambda_{j}+\bar{\mu}_{j}\right|}{\lambda_{j}}, \frac{2\left|\mu_{j}\right|}{\lambda_{j}}\right\} i\right)\right)}{2}\right) .
$$

The claim follows from the previous equations.

Now we estimate the term $V_{j}(s)$. 
Lemma 2.5. If $\sigma$ is a constant and $t$ is as in Lemma 2.4, then for all $j$

$$
\begin{aligned}
& \left|V_{j}(s)\right| \\
& \quad<\left|\frac{\left(\lambda_{j}+\bar{\mu}_{j}\right)^{2}}{\lambda_{j} t}\right|+2\left|\frac{\left(\lambda_{j}+\bar{\mu}_{j}\right)\left(\lambda_{j}+\bar{\mu}_{j}-\frac{1}{2}\right)}{\lambda_{j} t}\right|+\left|\frac{\mu_{j}^{2}}{\lambda_{j} t}\right|+2\left|\frac{\mu_{j}\left(\mu_{j}-\frac{1}{2}\right)}{\lambda_{j} t}\right| \\
& \quad+\frac{\left|B_{2}\right|}{2\left|\lambda_{j} s\right|}\left(2+\sec ^{2}\left(\frac{\arg \left(\lambda_{j}\left(-|\sigma|+\max _{j}\left\{\frac{2\left|\lambda_{j}+\bar{\mu}_{j}\right|}{\lambda_{j}}, \frac{2\left|\mu_{j}\right|}{\lambda_{j}}\right\} i\right)\right)}{2}\right)\right) .
\end{aligned}
$$

Proof. By the definition of the function $V_{j}(s)$ and the series expansion we have

$$
\begin{aligned}
V_{j}(s) & \left(-\lambda_{j} s+\lambda_{j}+\bar{\mu}_{j}-\frac{1}{2}\right) \log \left(1+\frac{\lambda_{j}+\bar{\mu}_{j}}{-\lambda_{j} s}\right)-\left(\lambda_{j}+\bar{\mu}_{j}\right) \\
& -\left(\lambda_{j} s+\mu_{j}-\frac{1}{2}\right) \log \left(1+\frac{\mu_{j}}{\lambda_{j} s}\right)+\mu_{j}+W\left(-\lambda_{j} s\right)-W\left(\lambda_{j} s\right) \\
= & \frac{\left(\lambda_{j}+\bar{\mu}_{j}\right)^{2}}{-\lambda_{j} s} \sum_{n=2}^{\infty} \frac{(-1)^{n+1}}{n}\left(\frac{\lambda_{j}+\bar{\mu}_{j}}{-\lambda_{j} s}\right)^{n-2} \\
& +\left(\lambda_{j}+\bar{\mu}_{j}-\frac{1}{2}\right) \sum_{n=1}^{\infty} \frac{(-1)^{n+1}}{n}\left(\frac{\lambda_{j}+\bar{\mu}_{j}}{-\lambda_{j} s}\right)^{n}-\frac{\mu_{j}^{2}}{\lambda_{j} s} \sum_{n=2}^{\infty} \frac{(-1)^{n+1}}{n}\left(\frac{\mu_{j}}{\lambda_{j} s}\right)^{n-2} \\
& -\left(\mu_{j}-\frac{1}{2}\right) \sum_{n=1}^{\infty} \frac{(-1)^{n+1}}{n}\left(\frac{\mu_{j}}{\lambda_{j} s}\right)^{n}+W\left(-\lambda_{j} s\right)-W\left(\lambda_{j} s\right) .
\end{aligned}
$$

Since $t \geq \max _{j}\left\{\frac{2\left|\lambda_{j}+\bar{\mu}_{j}\right|}{\lambda_{j}}\right\}$ and $t \geq \max _{j}\left\{\frac{2\left|\mu_{j}\right|}{\lambda_{j}}\right\}$, we have $\left|\frac{\lambda_{j}+\bar{\mu}_{j}}{-\lambda_{j} s}\right| \leq \frac{1}{2}$ and $\left|\frac{\mu_{j}}{\lambda_{j} s}\right| \leq \frac{1}{2}$ for all $j$. The claim follows from these estimates and Lemma 2.4 .

\section{The difference $I_{1}\left(T_{0}, T, b\right)-I_{1}\left(T_{0}, T, b+1\right)$}

In this section we estimate the integral

$$
I_{1}\left(T_{0}, T, b\right)-I_{1}\left(T_{0}, T, b+1\right)=\int_{T_{0}}^{T} \log |\mathcal{L}(b+i t)|-\log |\mathcal{L}(b+1+i t)| d t .
$$

\subsection{Preliminaries for the difference $I_{1}\left(T_{0}, T, b\right)-I_{1}\left(T_{0}, T, b+1\right)$}

In this section we prove preliminary results which are used to estimate the term $I_{1}\left(T_{0}, T, b\right)-I_{1}\left(T_{0}, T, b+1\right)$. The first one of these describes the properties of the logarithm. 
Lemma 3.1. Let $z$ be a complex number. Then

(a) if $|z|<\frac{1}{2}$ we have

$$
|\log (1+z)|<2|z|,
$$

(b) if $z$ is a real number we have

$$
|\log (1-z i)|<7|z|
$$

Proof. (a) Assume that $|z|<\frac{1}{2}$. By the series expansion of the logarithm we have

$$
|\log (1+z)|=\left|\sum_{n=1}^{\infty} \frac{(-1)^{n+1} z^{n}}{n}\right| \leq \sum_{n=1}^{\infty}\left|\frac{z^{n}}{n}\right| .
$$

Since $|z|<\frac{1}{2}$ and $n \geq 1$ in the sum, we have

$$
\sum_{n=1}^{\infty}\left|\frac{z^{n}}{n}\right|<\sum_{n=1}^{\infty}|z| \frac{1}{2^{n-1}}=2|z| .
$$

(b) Assume that $z$ is a real number. If $|z|<\frac{1}{2}$ then by the previous case we have $|\log (1-z i)|<2|z|$. Let $|z| \geq \frac{1}{2}$. Now we have

$$
|\log (1-z i)|^{2}=(\log |1-z i|)^{2}+(\arg (1-z i))^{2}<5|z|^{2}+4 \pi^{2}|z|^{2} .
$$

Thus

$$
|\log (1-z i)|<\sqrt{5+4 \pi^{2}}|z|<7|z|
$$

We use the previous lemma and basic properties of the absolute value to obtain the following two inequalities.

Lemma 3.2. If $\sigma<-3$ and $t>0$ then

$$
\begin{aligned}
& \mid \Re\left(\log \left(1-\frac{\sigma i}{t}\right)\left(d_{\mathcal{L}}\left(\frac{1}{2}-\sigma\right)+\frac{\Im(\mu) i}{2}\right)\right. \\
& \left.\quad-\log \left(1-\frac{(\sigma+1) i}{t}\right)\left(d_{\mathcal{L}}\left(-\frac{1}{2}-\sigma\right)+\frac{\Im(\mu) i}{2}\right)\right) \mid \\
& \quad<\frac{2}{t}\left|-d_{\mathcal{L}} \sigma+\frac{\Im(\mu) i}{2}\right|-7 \frac{d_{\mathcal{L}}}{2 t}(2 \sigma+1) .
\end{aligned}
$$


Proof. We have

$$
\begin{aligned}
& \mid \Re\left(\log \left(1-\frac{\sigma i}{t}\right)\left(d_{\mathcal{L}}\left(\frac{1}{2}-\sigma\right)+\frac{\Im(\mu) i}{2}\right)\right. \\
& \left.-\log \left(1-\frac{(\sigma+1) i}{t}\right)\left(d_{\mathcal{L}}\left(-\frac{1}{2}-\sigma\right)+\frac{\Im(\mu) i}{2}\right)\right) \mid \\
& \leq\left|\left(-d_{\mathcal{L}} \sigma+\frac{\Im(\mu) i}{2}\right) \log \left(1+\frac{i}{t-(\sigma+1) i}\right)\right| \\
& \quad+\left|\log \left(1-\frac{\sigma i}{t}\right) \frac{d_{\mathcal{L}}}{2}\right|+\left|\log \left(1-\frac{(\sigma+1) i}{t}\right) \frac{d_{\mathcal{L}}}{2}\right| .
\end{aligned}
$$

We want to estimate the previous terms. First we estimate the factor log $(1+$ $\left.\frac{i}{t-(\sigma+1) i}\right)$. Since $\sigma<-3$, we have

$$
\left|\frac{i}{t-(\sigma+1) i}\right|^{2}=\frac{1}{t^{2}+(\sigma+1)^{2}}<\frac{1}{4} .
$$

Thus $\left|\frac{i}{t-(\sigma+1) i}\right|<\frac{1}{2}$ and by Lemma 3.1 we get

$$
\begin{aligned}
& \left|\left(-d_{\mathcal{L}} \sigma+\frac{\Im(\mu) i}{2}\right) \log \left(1+\frac{i}{t-(\sigma+1) i}\right)\right| \\
& \quad<2\left|-d_{\mathcal{L}} \sigma+\frac{\Im(\mu) i}{2}\right| \frac{1}{|t-(\sigma+1) i|} .
\end{aligned}
$$

Further, by Lemma 3.1 we have

$$
\left|\log \left(1-\frac{\sigma i}{t}\right) \frac{d_{\mathcal{L}}}{2}\right|<-7 \frac{d_{\mathcal{L}} \sigma}{2 t}
$$

and

$$
\left|\log \left(1-\frac{(\sigma+1) i}{t}\right) \frac{d_{\mathcal{L}}}{2}\right|<-7 \frac{d_{\mathcal{L}}(\sigma+1)}{2 t} .
$$

The claim follows from the formulas (2), (3) , (4) and (5).

Lemma 3.3. If $|\sigma| \geq 1$ and $t>0$ then

$$
\begin{aligned}
& \left|\Re\left(-d_{\mathcal{L}}-d_{\mathcal{L}} \log \left(1-\frac{\sigma i}{t}\right) i t+d_{\mathcal{L}} \log \left(1-\frac{(\sigma+1) i}{t}\right) i t\right)\right| \\
& \quad<d_{\mathcal{L}}\left(\frac{3\left(\sigma^{2}+\sigma\right)}{t^{2}}+\frac{2}{t}\right) .
\end{aligned}
$$

Proof. We can calculate

$$
\begin{aligned}
\Re & \left(-d_{\mathcal{L}}-d_{\mathcal{L}} \log \left(1-\frac{\sigma i}{t}\right) i t+d_{\mathcal{L}} \log \left(1-\frac{(\sigma+1) i}{t}\right) i t\right) \\
& =-d_{\mathcal{L}}\left(1+t \arg \left(1-\frac{i}{t-\sigma i}\right)\right) .
\end{aligned}
$$


To obtain the absolute value of the term $-d_{\mathcal{L}}\left(1+t \arg \left(1-\frac{i}{t-\sigma i}\right)\right)$ we calculate the upper and lower bounds of this expression. We have $\arg \left(1-\frac{i}{t-\sigma i}\right)=$ $\arctan \left(\frac{-t}{t^{2}+\sigma^{2}+\sigma}\right)$. Thus $\arg \left(1-\frac{i}{t-\sigma i}\right) \leq 0$ and

$-d_{\mathcal{L}}\left(1+t \arg \left(1-\frac{i}{t-\sigma i}\right)\right)<-d_{\mathcal{L}}\left(1+\frac{-t^{2}}{t^{2}+\sigma^{2}+\sigma}\right) \leq-d_{\mathcal{L}}(1-1)=0$.

Next we compute the lower bound of the term $-d_{\mathcal{L}}\left(1+t \arg \left(1-\frac{i}{t-\sigma i}\right)\right)$.

By [15] we have $\arctan (x) \leq \frac{3 x}{1+2 \sqrt{1+x^{2}}}$ for $x \leq 0$. Thus

$$
\begin{aligned}
1+ & t \arctan \left(\frac{-t}{t^{2}+\sigma^{2}+\sigma}\right) \\
\leq 1- & \frac{3 t^{2}}{\left(t^{2}+\sigma^{2}+\sigma\right)\left(1+2 \sqrt{1+\left(\frac{-t}{t^{2}+\sigma^{2}+\sigma}\right)^{2}}\right)} .
\end{aligned}
$$

The right hand side is

$$
=\frac{t^{2}+\sigma^{2}+\sigma+2 \sqrt{\left(t^{2}+\sigma^{2}+\sigma\right)^{2}+t^{2}}-3 t^{2}}{t^{2}+\sigma^{2}+\sigma+2 \sqrt{\left(t^{2}+\sigma^{2}+\sigma\right)^{2}+t^{2}}} .
$$

Since

$$
t^{2}+\sigma^{2}+\sigma+2 \sqrt{\left(t^{2}+\sigma^{2}+\sigma\right)^{2}+t^{2}}-3 t^{2}<3\left(\sigma^{2}+\sigma\right)+2 t
$$

and

$$
t^{2}+\sigma^{2}+\sigma+2 \sqrt{\left(t^{2}+\sigma^{2}+\sigma\right)^{2}+t^{2}}>t^{2},
$$

by (6) and (7) we have

$$
1+t \arctan \left(\frac{-t}{t^{2}+\sigma^{2}+\sigma}\right)<\frac{3\left(\sigma^{2}+\sigma\right)}{t^{2}}+\frac{2}{t},
$$

as required.

Next we estimate two integrals. The estimates are used in the next section.

Theorem 3.4. Let $T_{0}$ and $T>T_{0}$ be positive real numbers. Then

$$
\left|\int_{T_{0}}^{T} \log \right| \mathcal{L}(1-b+i t)||<\frac{\pi^{2}}{3 \log 2} \text { and }\left|\int_{T_{0}}^{T} \log \right| \mathcal{L}(-b+i t)||<\frac{\pi^{2}}{3 \log 2} .
$$


Proof. We prove the claim only for the integral $\left|\int_{T_{0}}^{T} \log \right| \mathcal{L}(-b+i t) \mid$ since the other case can be proved similarly. First we look at the sum $\mathcal{L}(-b+$ $i t)-1=\sum_{n=2}^{\infty} \frac{a(n)}{n^{-b+i t}}$. By the Ramanujan hypothesis and the assumptions for the numbers $a_{1}$ and $b$

$$
\left|\sum_{n=2}^{\infty} \frac{a(n)}{n^{-b+i t}}\right| \leq \sum_{n=2}^{\infty} \frac{a_{1}}{n^{-b-1}}<1 .
$$

Thus we can use the Taylor series expansion of the logarithm of $\mathcal{L}(-b+i t)=$ $1+\sum_{n=2}^{\infty} \frac{a(n)}{n^{-b+i t}}$ and get

$$
\log |\mathcal{L}(-b+i t)|=\Re\left(\sum_{l=1}^{\infty} \frac{(-1)^{l}}{l} \sum_{n_{1}=2}^{\infty} \ldots \sum_{n_{l}=2}^{\infty} \frac{a\left(n_{1}\right) \cdots a\left(n_{l}\right)}{\left(n_{1} \cdots n_{l}\right)^{-b+i t}}\right) .
$$

We have

$$
\begin{aligned}
& \left|\int_{T_{0}}^{T} \log \right| \mathcal{L}(-b+i t)|| \\
& \quad=\left|\Re\left(\sum_{l=1}^{\infty} \frac{(-1)^{l}}{l} \sum_{n_{1}=2}^{\infty} \cdots \sum_{n_{l}=2}^{\infty} \frac{a\left(n_{1}\right) \cdots a\left(n_{l}\right)}{\left(n_{1} \cdots n_{l}\right)^{-b}} \int_{T_{0}}^{T} \frac{d t}{\left(n_{1} \cdots n_{l}\right)^{i t}}\right)\right|
\end{aligned}
$$

By the Ramanujan hypothesis

$$
\begin{aligned}
& \left|\Re\left(\sum_{l=1}^{\infty} \frac{(-1)^{l}}{l} \sum_{n_{1}=2}^{\infty} \ldots \sum_{n_{l}=2}^{\infty} \frac{a\left(n_{1}\right) \cdots a\left(n_{l}\right)}{\left(n_{1} \cdots n_{l}\right)^{-b}} \int_{T_{0}}^{T} \frac{d t}{\left(n_{1} \cdots n_{l}\right)^{i t}}\right)\right| \\
& \quad \leq \sum_{l=1}^{\infty} \frac{1}{l} \sum_{n_{1}=2}^{\infty} \ldots \sum_{n_{l}=2}^{\infty} \frac{a_{1}^{l}}{\left(n_{1} \cdots n_{l}\right)^{-b-1}}\left|\int_{T_{0}}^{T} \frac{d t}{\left(n_{1} \cdots n_{l}\right)^{i t}}\right| .
\end{aligned}
$$

For $n \geq 2^{l}$ we have

$$
\left|\int_{T_{0}}^{T} \frac{d t}{n^{i t}}\right|=\left|\frac{i}{\log n}\left(e^{-i T \log n}-e^{-i T_{0} \log n}\right)\right| \leq \frac{2}{l \log 2} .
$$

Thus

$$
\begin{aligned}
& \sum_{l=1}^{\infty} \frac{1}{l} \sum_{n_{1}=2}^{\infty} \cdots \sum_{n_{l}=2}^{\infty} \frac{a_{1}^{l}}{\left(n_{1} \cdots n_{l}\right)^{-b-1}}\left|\int_{T_{0}}^{T} \frac{d t}{\left(n_{1} \cdots n_{l}\right)^{i t}}\right| \\
& \quad \leq \sum_{l=1}^{\infty} \frac{2}{l^{2} \log 2}\left(\sum_{n=2}^{\infty} \frac{a_{1}}{n^{-b-1}}\right)^{l} .
\end{aligned}
$$


Since we assume that $\sum_{n=2}^{\infty} \frac{a_{1}}{n^{-b-1}}<1$, we have

$$
\sum_{l=1}^{\infty} \frac{2}{l^{2} \log 2}\left(\sum_{n=2}^{\infty} \frac{a_{1}}{n^{-b-1}}\right)^{l}<\sum_{l=1}^{\infty} \frac{2}{l^{2} \log 2}=\frac{\pi^{2}}{3 \log 2} .
$$

\subsection{The estimate of the difference $I_{1}\left(T_{0}, T, b\right)-I_{1}\left(T_{0}, T, b+1\right)$}

In this section we estimate the term

$$
\left|I_{1}\left(T_{0}, T, b\right)-I_{1}\left(T_{0}, T, b+1\right)-\int_{T_{0}}^{T} d_{\mathcal{L}} \log t+\log \left(\lambda Q^{2}\right) d t\right|
$$

which is used to get the main result. We obtain the estimate by using the results which we have obtained in Sections 2 and 3.1. We want to simplify the notation and thus we define for real numbers $T_{0}$ and $T$

$$
\begin{aligned}
& S_{j}\left(T_{0}, T, b\right) \\
& =\frac{2}{\lambda_{j}} \log \frac{T}{T_{0}}\left(\left|\lambda_{j}+\bar{\mu}_{j}\right|^{2}+2\left|\left(\lambda_{j}+\bar{\mu}_{j}\right)\left(\lambda_{j}+\bar{\mu}_{j}-\frac{1}{2}\right)\right|+\left|\mu_{j}\right|^{2}+2\left|\mu_{j}\left(\mu_{j}-\frac{1}{2}\right)\right|\right. \\
& +\frac{\left|B_{2}\right|}{4}\left(2+\sec ^{2}\left(\frac{\arg \left(\lambda_{j}\left(-b+\max _{j}\left\{\frac{2\left|\lambda_{j}+\bar{\mu}_{j}\right|}{\lambda_{j}}, \frac{2\left|\mu_{j}\right|}{\lambda_{j}}\right\} i\right)\right)}{2}\right)\right) \\
& \left.+\frac{\left|B_{2}\right|}{4}\left(2+\sec ^{2}\left(\frac{\arg \left(\lambda_{j}\left(-b-1+\max _{j}\left\{\frac{2\left|\lambda_{j}+\bar{\mu}_{j}\right|}{\lambda_{j}}, \frac{2\left|\mu_{j}\right|}{\lambda_{j}}\right\} i\right)\right)}{2}\right)\right)\right) \\
& S\left(T_{0}, T, b\right)=\sum_{j=1}^{f} S_{j}\left(T_{0}, T, b\right) \text { and } \\
& R_{1}\left(T_{0}, T, b\right)=\log \frac{T}{T_{0}}\left(-7 \frac{d_{\mathcal{L}}}{2}(2 b+1)+2\left|-d_{\mathcal{L}} b+\frac{\Im(\mu) i}{2}\right|+2 d_{\mathcal{L}}\right) \\
& \quad+\frac{3 d_{\mathcal{L}}\left(b^{2}+b\right)}{T_{0}}+S\left(T_{0}, T, b\right) .
\end{aligned}
$$

Theorem 3.5. If $T_{0} \geq \max _{j}\left\{\frac{2\left|\lambda_{j}+\bar{\mu}_{j}\right|}{\lambda_{j}}\right\}, T_{0}>\max _{j}\left\{\frac{2\left|\mu_{j}\right|}{\lambda_{j}}\right\}$ and $T>T_{0}$ then

$$
\begin{aligned}
& \left|I_{1}\left(T_{0}, T, b\right)-I_{1}\left(T_{0}, T, b+1\right)-\int_{T_{0}}^{T} d_{\mathcal{L}} \log t+\log \left(\lambda Q^{2}\right) d t\right| \\
& \quad<R_{1}\left(T_{0}, T, b\right)+\frac{2 \pi^{2}}{3 \log 2} .
\end{aligned}
$$


Proof. By the definition of the integral $I_{1}\left(T_{0}, T, b\right)$ and since $\mathcal{L}(s)=$ $\Delta_{\mathcal{L}}(s) \overline{\mathcal{L}(1-\bar{s})}$, we have

$$
\begin{aligned}
& I_{1}\left(T_{0}, T, b\right)-I_{1}\left(T_{0}, T, b+1\right) \\
& =\int_{T_{0}}^{T} \log \left|\Delta_{\mathcal{L}}(b+i t)\right|-\log \left|\Delta_{\mathcal{L}}(b+1+i t)\right| d t \\
& \quad+\int_{T_{0}}^{T} \log |\mathcal{L}(1-b+i t)|-\log |\mathcal{L}(-b+i t)| d t .
\end{aligned}
$$

We will first estimate the terms and the claim follows when we sum the estimates.

First we look at the part $\log \left|\Delta_{\mathcal{L}}(b+i t)\right|-\log \left|\Delta_{\mathcal{L}}(b+1+i t)\right|$ and its integral. Since $T_{0}>\max _{j}\left\{\frac{2\left|\mu_{j}\right|}{\lambda_{j}}\right\}$, for $t \geq T_{0}$ it holds that

$$
\left\{\begin{array}{l}
\left|\arg \left(\lambda_{j}(1-b-i t)+\bar{\mu}_{j}\right)\right|<\pi \\
\left|\arg \left(\lambda_{j}(b+i t)+\mu_{j}\right)\right|<\pi \\
\left|\arg \left(\lambda_{j}(-b-i t)+\bar{\mu}_{j}\right)\right|<\pi \text { and } \\
\left|\arg \left(\lambda_{j}(b+1+i t)+\mu_{j}\right)\right|<\pi
\end{array} .\right.
$$

Thus by Lemma 2.3 we have

$$
\begin{aligned}
& \log \left|\Delta_{\mathcal{L}}(b+i t)\right|-\log \left|\Delta_{\mathcal{L}}(b+1+i t)\right|=\left(d_{\mathcal{L}} \log t+\log \left(\lambda Q^{2}\right)\right)-d_{\mathcal{L}}+\Re(V(b+i t)-V(b+1+i t)) \\
& \quad+\Re\left(\log \left(1-\frac{b i}{t}\right)\left(d_{\mathcal{L}}\left(\frac{1}{2}-b-i t\right)+\frac{\Im(\mu) i}{2}\right)\right. \\
&\left.\quad-\log \left(1-\frac{(b+1) i}{t}\right)\left(d_{\mathcal{L}}\left(-\frac{1}{2}-b-i t\right)+\frac{\Im(\mu) i}{2}\right)\right) .
\end{aligned}
$$

Now we estimate the last difference between the last term and the term $d_{\mathcal{L}}$. By Lemma 3.2 and Lemma 3.3 we have

$$
\begin{aligned}
& \mid \Re\left(\log \left(1-\frac{b i}{t}\right)\left(d_{\mathcal{L}}\left(\frac{1}{2}-b-i t\right)+\frac{\Im(\mu) i}{2}\right)\right. \\
& \left.\quad-\log \left(1-\frac{(b+1) i}{t}\right)\left(d_{\mathcal{L}}\left(-\frac{1}{2}-b-i t\right)+\frac{\Im(\mu) i}{2}\right)-d_{\mathcal{L}}\right) \mid \\
& \quad<\frac{2}{t}\left|-d_{\mathcal{L}} b+\frac{\Im(\mu) i}{2}\right|-7 \frac{d_{\mathcal{L}}}{2 t}(2 b+1)+d_{\mathcal{L}}\left(\frac{3\left(b^{2}+b\right)}{t^{2}}+\frac{2}{t}\right) .
\end{aligned}
$$


We can integrate this and get

$$
\begin{aligned}
& \int_{T_{0}}^{T} \frac{2}{t}\left|-d_{\mathcal{L}} b+\frac{\Im(\mu) i}{2}\right|-7 \frac{d_{\mathcal{L}}}{2 t}(2 b+1)+d_{\mathcal{L}}\left(\frac{3\left(b^{2}+b\right)}{t^{2}}+\frac{2}{t}\right) d t \\
& \quad<\log \frac{T}{T_{0}}\left(-7 \frac{d_{\mathcal{L}}}{2}(2 b+1)+2\left|-d_{\mathcal{L}} b+\frac{\Im(\mu) i}{2}\right|+2 d_{\mathcal{L}}\right)+\frac{3 d_{\mathcal{L}}\left(b^{2}+b\right)}{T_{0}} .
\end{aligned}
$$

Further, we can estimate the integral of the term $\Re(V(b+i t)-V(b+1+i t))$. We remember that $V(s)=\sum_{j=1}^{f} V_{j}(s)$. By Lemma 2.5

$$
\begin{aligned}
& \left|V_{j}(b+i t)\right|+\left|V_{j}(b+1+i t)\right| \\
& <2\left|\frac{\left(\lambda_{j}+\bar{\mu}_{j}\right)^{2}}{\lambda_{j} t}\right|+4\left|\frac{\left(\lambda_{j}+\bar{\mu}_{j}\right)\left(\lambda_{j}+\bar{\mu}_{j}-\frac{1}{2}\right)}{\lambda_{j} t}\right|+2\left|\frac{\mu_{j}^{2}}{\lambda_{j} t}\right|+4\left|\frac{\mu_{j}\left(\mu_{j}-\frac{1}{2}\right)}{\lambda_{j} t}\right| \\
& \quad+\frac{\left|B_{2}\right|}{2\left|\lambda_{j} t\right|}\left(2+\sec ^{2}\left(\frac{\arg \left(\lambda_{j}\left(-b+\max _{j}\left\{\frac{2\left|\lambda_{j}+\bar{\mu}_{j}\right|}{\lambda_{j}}, \frac{2\left|\mu_{j}\right|}{\lambda_{j}}\right\} i\right)\right)}{2}\right)\right) \\
& \quad+\frac{\left|B_{2}\right|}{2\left|\lambda_{j} t\right|}\left(2+\sec ^{2}\left(\frac{\arg \left(\lambda_{j}\left(-b-1+\max _{j}\left\{\frac{2\left|\lambda_{j}+\bar{\mu}_{j}\right|}{\lambda_{j}}, \frac{2\left|\mu_{j}\right|}{\lambda_{j}}\right\} i\right)\right)}{2}\right)\right) .
\end{aligned}
$$

We can sum these terms, integrate and get

$$
\left|\int_{T_{0}}^{T} \Re(V(b+i t)-V(b+1+i t)) d t\right|<S\left(T_{0}, T, b\right) .
$$

By Theorem 3.4

$$
\left|\int_{T_{0}}^{T} \log \right| \mathcal{L}(1-b+i t)|-\log | \mathcal{L}(-b+i t)|d t|<\frac{2 \pi^{2}}{3 \log 2} .
$$

The claim follows immediately when we sum the estimates (9), (10), (111) and (12) together.

\section{Integral $I_{3}(T, a, b)$}

In this section we estimate the integral $I_{3}(T, a, b)=\int_{b}^{a} \arg \mathcal{L}(\sigma+i T) d \sigma$.

\subsection{Preliminaries for the integral $I_{3}(T, a, b)$}

In this section we collect some preliminary results which are needed in the estimates of the integral $I_{3}(T, a, b)$. Our first goal is to estimate the function $\mathcal{L}(s)$, and to do this, we apply the Phragmén-Lindelöf principle for a strip. We also need the following lemma. 
Lemma 4.1. If $t \geq 1$ then

$$
\Re\left(\log \left(1+\frac{2 i}{t}\right)\left(d_{\mathcal{L}}\left(\frac{5}{2}-i t\right)+\frac{\Im(\mu) i}{2}\right)\right)<\frac{5 \sqrt{5}+4}{2} d_{\mathcal{L}}+|\Im(\mu)| .
$$

Proof. Since $t \geq 1$, we get

$$
\begin{aligned}
\Re & \left(\log \left(1+\frac{2 i}{t}\right)\left(d_{\mathcal{L}}\left(\frac{5}{2}-i t\right)+\frac{\Im(\mu) i}{2}\right)\right) \\
& =\log \left|1+\frac{2 i}{t}\right| \frac{5}{2} d_{\mathcal{L}}+\arg \left(1+\frac{2 i}{t}\right) d_{\mathcal{L}} t-\arg \left(1+\frac{2 i}{t}\right) \frac{\Im(\mu)}{2} \\
& <\left|1+\frac{2 i}{t}\right| \frac{5}{2} d_{\mathcal{L}}+\left|\arctan \left(\frac{2}{t}\right) d_{\mathcal{L}} t\right|+\left|\arctan \left(\frac{2}{t}\right) \frac{\Im(\mu)}{2}\right| \\
& <\frac{5}{2} \sqrt{1^{2}+2^{2}} d_{\mathcal{L}}+\frac{2}{t} d_{\mathcal{L}} t+\frac{2}{t}\left|\frac{\Im(\mu)}{2}\right| \\
& \leq \frac{5 \sqrt{5}+4}{2} d_{\mathcal{L}}+|\Im(\mu)| .
\end{aligned}
$$

We denote $R=a-b$. Note that $R>0$ and $a-2 R<0$. Let

$$
\begin{aligned}
M_{1}= & \max \left\{3^{k} \frac{a_{1} \pi^{2}}{6}, 3^{k} \frac{a_{1} \pi^{2}}{6}\left|\lambda Q^{2}\right|^{\frac{5}{2}} \exp \left(\frac{5 \sqrt{5}}{2} d_{\mathcal{L}}+|\Im(\mu)|+\frac{1}{\left|\lambda_{j}(T-2 R)\right|} \times\right.\right. \\
& \times \sum_{j=1}^{f}\left(\left|\lambda_{j}+\bar{\mu}_{j}\right|^{2}+2\left|\left(\lambda_{j}+\bar{\mu}_{j}\right)\left(\lambda_{j}+\bar{\mu}_{j}-\frac{1}{2}\right)\right|+\left|\mu_{j}\right|^{2}+2\left|\mu_{j}\left(\mu_{j}-\frac{1}{2}\right)\right|\right. \\
& \left.\left.\left.+\frac{\left|B_{2}\right|}{2}\left(2+\sec ^{2}\left(\frac{\arg \left(\lambda_{j}\left(-2+\max _{j}\left\{\frac{2\left|\lambda_{j}+\bar{\mu}_{j}\right|}{\lambda_{j}}, \frac{2\left|\mu_{j}\right|}{\lambda_{j}}\right\} i\right)\right)}{2}\right)\right)\right)\right)\right\} .
\end{aligned}
$$

Let also $k$ be defined as in the condition 2 for Selberg class. By using the Phragmén-Lindelöf principle for a strip we get the following theorem.

Theorem 4.2. Let

$$
T \geq\left\{\begin{array}{l}
2 R+1 \\
2 R+\max _{j}\left\{\frac{2\left|\lambda_{j}+\bar{\mu}_{j}\right|}{\lambda_{j}}\right\} \\
2 R+\frac{1}{2^{\frac{1}{k}}-1}, \text { if } k>0
\end{array},\right.
$$


$T>2 R+\max _{j}\left\{\frac{2\left|\mu_{j}\right|}{\lambda_{j}}\right\}$ and $t \in[T-2 R, T+2 R]$. Then $|\mathcal{L}(s)| \leq \frac{a_{1} \pi^{2}}{6}$, if $\sigma \geq 3$

$$
\begin{aligned}
|\mathcal{L}(s)| \leq & t^{d_{\mathcal{L}}\left(\frac{1}{2}-\sigma\right)} \frac{a_{1} \pi^{2}}{6}\left|\lambda Q^{2}\right|^{\frac{1}{2}-\sigma} \times \\
& \times \exp \left(d_{\mathcal{L}} \sigma+\Re\left(\log \left(1-\frac{\sigma i}{t}\right)\left(d_{\mathcal{L}}\left(\frac{1}{2}-s\right)+\frac{\Im(\mu) i}{2}\right)+V(s)\right)\right),
\end{aligned}
$$

if $\sigma \leq-2$ and $|\mathcal{L}(s)|<2^{\frac{5}{2} d_{\mathcal{L}}+1} M_{1} t^{\frac{1}{2} d_{\mathcal{L}}(3-\sigma)}$ if $-2 \leq \sigma \leq 3$.

Proof. We prove the claim in the different parts depending on the value of the real number $\sigma$. Assume $\sigma \geq 3$. Then

$$
|\mathcal{L}(s)|=\left|\sum_{n=1}^{\infty} \frac{a(n)}{n^{s}}\right| \leq \sum_{n=1}^{\infty} \frac{a_{1}}{n^{2}}=\frac{a_{1} \pi^{2}}{6} .
$$

Next we look at the case $\sigma \leq-2$. We have $\mathcal{L}(s)=\Delta_{\mathcal{L}}(s) \overline{\mathcal{L}(1-\bar{s})}$ and by the previous case $|\overline{\mathcal{L}(1-\bar{s})}| \leq \frac{a_{1} \pi^{2}}{6}$. Since $t>\max _{j}\left\{\frac{2\left|\mu_{j}\right|}{\lambda_{j}}\right\}$, it holds that $\left|\arg \left(\lambda_{j}(1-s)+\bar{\mu}_{j}\right)\right|<\pi,\left|\arg \left(\lambda_{j} s+\mu_{j}\right)\right|<\pi$ and $t>0$. Thus by Lemma 2.3 for $\sigma \leq-2$ we have

$$
\begin{aligned}
|\mathcal{L}(s)| \leq & t^{d_{\mathcal{L}}\left(\frac{1}{2}-\sigma\right)} \frac{a_{1} \pi^{2}}{6}\left|\lambda Q^{2}\right|^{\frac{1}{2}-\sigma} \times \\
& \times \exp \left(d_{\mathcal{L}} \sigma+\Re\left(\log \left(1-\frac{\sigma i}{t}\right)\left(d_{\mathcal{L}}\left(\frac{1}{2}-s\right)+\frac{\Im(\mu) i}{2}\right)+V(s)\right)\right) .
\end{aligned}
$$

Now we look at the case $-2 \leq \sigma \leq 3$. First we look at the function $(s-1)^{k} \mathcal{L}(s)$. Since $(s-1)^{k} \mathcal{L}(s)$ is an analytic function of the finite order, we can apply the Phragmén-Lindelöf principle for a strip [11, Theorem 5.53]. By the case $\sigma \geq 3$ and since $t>0$, we have

$$
|\mathcal{L}(3+i t)| \leq \frac{a_{1} \pi^{2}}{6}=\frac{a_{1} \pi^{2}}{6}(1+t)^{0} .
$$

Further, by the case $\sigma \leq-2$ and Lemma 4.1 we have

$$
\begin{aligned}
& |\mathcal{L}(-2+i t)| \\
& \leq t^{d_{\mathcal{L}}\left(\frac{1}{2}+2\right)} \frac{a_{1} \pi^{2}}{6}\left|\lambda Q^{2}\right|^{\frac{1}{2}+2} \exp \left(-2 d_{\mathcal{L}}\right. \\
& \left.+\Re\left(\log \left(1+\frac{2 i}{t}\right)\left(d_{\mathcal{L}}\left(\frac{1}{2}+2-i t\right)+\frac{\Im(\mu) i}{2}\right)+V(-2+i t)\right)\right) \\
& <(1+t)^{\frac{5}{2}} d_{\mathcal{L}} \frac{a_{1} \pi^{2}}{6}\left|\lambda Q^{2}\right|^{\frac{5}{2}} \exp \left(\frac{5 \sqrt{5}}{2} d_{\mathcal{L}}+|\Im(\mu)|+\sup _{t \in[T-2 R, T+2 R]}|V(-2+i t)|\right) .
\end{aligned}
$$


Also, from Lemma 2.5 it follows that

$$
\begin{aligned}
\sup _{t \in[T-2 R, T+2 R]}|V(-2+i t)| \\
<\frac{1}{\left|\lambda_{j}(T-2 R)\right|} \sum_{j=1}^{f}\left(\left|\lambda_{j}+\bar{\mu}_{j}\right|^{2}\right. \\
\quad+2\left|\left(\lambda_{j}+\bar{\mu}_{j}\right)\left(\lambda_{j}+\bar{\mu}_{j}-\frac{1}{2}\right)\right|+\left|\mu_{j}\right|^{2}+2\left|\mu_{j}\left(\mu_{j}-\frac{1}{2}\right)\right| \\
\left.\quad+\frac{\left|B_{2}\right|}{2}\left(2+\sec ^{2}\left(\frac{\arg \left(\lambda_{j}\left(-2+\max _{j}\left\{\frac{2\left|\lambda_{j}+\bar{\mu}_{j}\right|}{\lambda_{j}}, \frac{2\left|\mu_{j}\right|}{\lambda_{j}}\right\} i\right)\right)}{2}\right)\right)\right) .
\end{aligned}
$$

Let $l(x)=-\frac{5}{x}+\frac{3}{5}$. We estimate the function $(s-1)^{k} \mathcal{L}(s)$ in two different cases; $k=0$ and $k>0$.

First we look at the case $k=0$. This means that $(s-1)^{k} \mathcal{L}(s)=\mathcal{L}(s)$. Let

$$
M_{\sigma_{1}}=\frac{a_{1} \pi^{2}}{6}\left|\lambda Q^{2}\right|^{\frac{5}{2}} \exp \left(\frac{5 \sqrt{5}}{2} d_{\mathcal{L}}+|\Im(\mu)|+\sup _{t \in[T-2 R, T+2 R]}|V(-2+i t)|\right) .
$$

and $M_{\sigma_{2}}=\frac{a_{1} \pi^{2}}{6}$. By the Phragmén-Lindelöf principle for a strip for $-2 \leq$ $\sigma \leq 3$ and the inequality (13) we have

$$
|\mathcal{L}(s)| \leq M_{\sigma_{1}}^{l(\sigma)} M_{\sigma_{2}}^{1-l(\sigma)}(1+t)^{l(\sigma) \frac{5}{2} d_{\mathcal{L}}}<M_{1}(1+t)^{\frac{1}{2} d_{\mathcal{L}}(3-\sigma)} .
$$

Now we look at the case $k>0$. Since $k$ is an integer, we have $k \geq 1$. By triangle inequality for $\sigma \in[-2,3]$ we have

$$
|s-1|^{k} \leq(|\sigma-1|+t)^{k} \leq 3^{k}\left(1+\frac{t}{3}\right)^{k}<3^{k}(1+t)^{k} .
$$

This inequality is used when we apply the Phragmén-Lindelöf principle for a strip. Let

$$
M_{\sigma_{1}}=3^{k} \frac{a_{1} \pi^{2}}{6}\left|\lambda Q^{2}\right|^{\frac{5}{2}} \exp \left(\frac{5 \sqrt{5}}{2} d_{\mathcal{L}}+|\Im(\mu)|+\sup _{t \in[T-2 R, T+2 R]}|V(-2+i t)|\right)
$$

and $M_{\sigma_{2}}=3^{k} \frac{a_{1} \pi^{2}}{6}$. By the Phragmén-Lindelöf principle for a strip in $-2 \leq$ $\sigma \leq 3$, the inequality (13) and the inequality (15) we have

$\left|(s-1)^{k} \mathcal{L}(s)\right| \leq M_{\sigma_{1}}^{l(\sigma)} M_{\sigma_{2}}^{1-l(\sigma)}(1+t)^{l(\sigma)\left(k+\frac{5}{2} d_{\mathcal{L}}\right)+k(1-l(\sigma))}<M_{1}(1+t)^{\frac{1}{2} d_{\mathcal{L}}(3-\sigma)+k}$. 
Thus $|\mathcal{L}(s)|<M_{1}\left(\frac{1+t}{|s-1|}\right)^{k}(1+t)^{\frac{1}{2} d_{\mathcal{L}}(3-\sigma)}$. Since by assumptions $t \in[T-$ $2 R, T+2 R]$, we have $t \geq \frac{1}{2^{\frac{1}{k}}-1}$. Thus

$$
M_{1}\left(\frac{1+t}{|s-1|}\right)^{k}(1+t)^{\frac{1}{2} d_{\mathcal{L}}(3-\sigma)} \leq 2 M_{1}(1+t)^{\frac{1}{2} d_{\mathcal{L}}(3-\sigma)} .
$$

By the inequalities (14) and (16) we have

$$
|\mathcal{L}(s)|<M_{1}(1+t)^{\frac{1}{2} d_{\mathcal{L}}(3-\sigma)}<2 M_{1}(1+t)^{\frac{1}{2} d_{\mathcal{L}}(3-\sigma)}
$$

if $k=0$ and

$$
|\mathcal{L}(s)|<2 M_{1}(1+t)^{\frac{1}{2}} d_{\mathcal{L}}(3-\sigma)
$$

if $k>0$. Further, for $\sigma \in[-2,3]$ and $t \geq 1$ we have

$$
(1+t)^{\frac{1}{2} d_{\mathcal{L}}(3-\sigma)} \leq(2 t)^{\frac{1}{2} d_{\mathcal{L}}(3-\sigma)} \leq 2^{\frac{5}{2} d_{\mathcal{L}}} t^{\frac{1}{2} d_{\mathcal{L}}(3-\sigma)} .
$$

Thus

$$
|\mathcal{L}(s)|<2^{\frac{5}{2} d_{\mathcal{L}}+1} M_{1} t^{\frac{1}{2} d_{\mathcal{L}}(3-\sigma)}
$$

for all $k$.

The following property is also useful in estimating the integral $I_{3}(T, a, b)$.

Lemma 4.3. Let $\sigma \in[a-2 R, a+2 R], T>2 R$ and $t \in[T-2 R, T+2 R]$. Then

$$
\begin{aligned}
& \Re\left(\log \left(1-\frac{\sigma i}{t}\right)\left(d_{\mathcal{L}}\left(\frac{1}{2}-s\right)+\frac{\Im(\mu) i}{2}\right)\right) \\
& \quad<\left|1-\frac{(a+2 R) i}{T-2 R}\right| d_{\mathcal{L}}\left(\frac{1}{2}-a+2 R\right)+d_{\mathcal{L}}(a+2 R)+\frac{a+2 R}{T-2 R}\left|\frac{\Im(\mu)}{2}\right| .
\end{aligned}
$$

Proof. We have

$$
\begin{aligned}
& \Re\left(\log \left(1-\frac{\sigma i}{t}\right)\left(d_{\mathcal{L}}\left(\frac{1}{2}-s\right)+\frac{\Im(\mu) i}{2}\right)\right) \\
& \quad=\log \left|1-\frac{\sigma i}{t}\right| d_{\mathcal{L}}\left(\frac{1}{2}-\sigma\right)+\arg \left(1-\frac{\sigma i}{t}\right)\left(d_{\mathcal{L}} t-\frac{\Im(\mu)}{2}\right) .
\end{aligned}
$$

By the assumptions for the numbers $\sigma$ and $t$ we have $|\sigma| \leq a+2 R$ and $t \geq T-2 R>0$. Thus

$$
\log \left|1-\frac{\sigma i}{t}\right| d_{\mathcal{L}}\left(\frac{1}{2}-\sigma\right)<\left|1-\frac{(a+2 R) i}{T-2 R}\right| d_{\mathcal{L}}\left(\frac{1}{2}-a+2 R\right)
$$

and

$$
\arg \left(1-\frac{\sigma i}{t}\right)\left(d_{\mathcal{L}} t-\frac{\Im(\mu)}{2}\right)<d_{\mathcal{L}}(a+2 R)+\frac{a+2 R}{T-2 R}\left|\frac{\Im(\mu)}{2}\right| .
$$

The claim follows immediately from the formulas (17), (18) and (19). 


\subsection{The estimate of the integral $I_{3}(T, a, b)$}

In this section we estimate the integral $I_{3}(T, a, b)$. Since the estimate contains the term $V(s)$, where $\sigma$ and $t$ lie in specific intervals, we also need estimate the term $V(s)$ on these intervals. We want to shorten our notation and thus we define the following terms: Let $T$ be a real number,

$V^{*}(T)$

$$
\begin{aligned}
= & \frac{1}{T-2 R} \sum_{j=1}^{f} \frac{1}{\lambda_{j}}\left(\left|\lambda_{j}+\bar{\mu}_{j}\right|^{2}\right. \\
& +2\left|\left(\lambda_{j}+\bar{\mu}_{j}\right)\left(\lambda_{j}+\bar{\mu}_{j}-\frac{1}{2}\right)\right|+\left|\mu_{j}\right|^{2}+2\left|\mu_{j}\left(\mu_{j}-\frac{1}{2}\right)\right| \\
& \left.+\frac{\left|B_{2}\right|}{2}\left(2+\sec ^{2}\left(\frac{\arg \left(\lambda_{j}\left(-a-2 R+\max _{j}\left\{\frac{2\left|\lambda_{j}+\bar{\mu}_{j}\right|}{\lambda_{j}}, \frac{2\left|\mu_{j}\right|}{\lambda_{j}}\right\} i\right)\right)}{2}\right)\right)\right)
\end{aligned}
$$

and

$$
\begin{aligned}
R_{2}(T) & \frac{1}{\log 2}\left(d_{\mathcal{L}}\left(\frac{1}{2}-a+2 R\right) \log (2 T)+\log \frac{a_{1} \pi^{2}}{6}+V^{*}(T)\right. \\
& +\max \left\{\max \left\{\frac{5}{2} \log \left|\lambda Q^{2}\right|,\left(\frac{1}{2}-a+2 R\right) \log \left|\lambda Q^{2}\right|\right\}-2 d_{\mathcal{L}}\right. \\
& +\left|1-\frac{(a+2 R) i}{T-2 R}\right| d_{\mathcal{L}}\left(\frac{1}{2}-a+2 R\right)+d_{\mathcal{L}}(a+2 R)+\frac{a+2 R}{T-2 R}\left|\frac{\Im(\mu)}{2}\right|, \\
& \left.\left.\left(\frac{5}{2} d_{\mathcal{L}}+1\right) \log 2+k \log 3+\max \left\{0, \frac{5}{2} \log \left|\lambda Q^{2}\right|+\frac{5 \sqrt{5}}{2} d_{\mathcal{L}}+|\Im(\mu)|\right\}\right\}\right)
\end{aligned}
$$

Lemma 4.4. Assume that $T$ satisfies the same conditions as in Theorem 4.2, Let

$$
g(z)=\frac{1}{2}(\mathcal{L}(z+i T)+\overline{\mathcal{L}(\bar{z}+i T)}) .
$$

Then

$$
\left|\frac{1}{2 \pi \log 2} \int_{0}^{2 \pi} \log \right| g\left(a+2 R e^{i \theta}\right)|d \theta|<R_{2}(T)
$$

if $\int_{0}^{2 \pi} \log \left|g\left(a+2 R e^{i \theta}\right)\right| d \theta \geq 0$.

Proof. We assume that $\int_{0}^{2 \pi} \log \left|g\left(a+2 R e^{i \theta}\right)\right| d \theta \geq 0$. First we estimate the term $\left|g\left(a+2 R e^{i \theta}\right)\right|$. To do this we estimate the functions $\mathcal{L}(z+i T)$ and 
$\overline{\mathcal{L}(\bar{z}+i T)}$ by Theorem 4.2 and Lemma 4.3 . First we define the function $M(T)$. Let

$$
\begin{aligned}
M(T)= & \frac{a_{1} \pi^{2}}{6} \max \left\{| \lambda Q ^ { 2 } | ^ { \frac { 5 } { 2 } } \operatorname { e x p } \left(-2 d_{\mathcal{L}}+\left|1-\frac{(a+2 R) i}{T-2 R}\right| d_{\mathcal{L}}\left(\frac{1}{2}-a+2 R\right)\right.\right. \\
& \left.+d_{\mathcal{L}}(a+2 R)+\frac{a+2 R}{T-2 R}\left|\frac{\Im(\mu)}{2}\right|\right),\left|\lambda Q^{2}\right|^{\frac{1}{2}-a+2 R} \exp \left(-2 d_{\mathcal{L}}\right. \\
& \left.+\left|1-\frac{(a+2 R) i}{T-2 R}\right| d_{\mathcal{L}}\left(\frac{1}{2}-a+2 R\right)+d_{\mathcal{L}}(a+2 R)+\frac{a+2 R}{T-2 R}\left|\frac{\Im(\mu)}{2}\right|\right), \\
& \left.2^{\frac{5}{2} d_{\mathcal{L}}+1} 3^{k}, 2^{\frac{5}{2} d_{\mathcal{L}}+1} 3^{k}\left|\lambda Q^{2}\right|^{\frac{5}{2}} \exp \left(\frac{5 \sqrt{5}}{2} d_{\mathcal{L}}+|\Im(\mu)|\right)\right\} .
\end{aligned}
$$

Since the estimates of the functions $\mathcal{L}(z+i T)$ and $\overline{\mathcal{L}(\bar{z}+i T)}$ contain restrictions of the imaginary and real parts, we estimate them. We have

$$
\Re\left(a+2 R e^{ \pm i \theta}+i T\right)=a+2 R \cos (\theta) \in[a-2 R, a+2 R] .
$$

Further, we have

$$
\left|\Im\left(a+2 R e^{ \pm i \theta}+i T\right)\right|=|2 R \sin ( \pm \theta)+T| \in[T-2 R, T+2 R] .
$$

Note that $[-2,3] \subset[a-2 R, a+2 R]$. Further, note that by Lemma 2.5

$$
\sup _{\sigma \in[a-2 R, a+2 R], t \in[T-2 R, T+2 R]}|V(s)|<V^{*}(T) .
$$

Thus by Theorem 4.2 and Lemma 4.3

$$
\left|\mathcal{L}\left(a+2 R e^{ \pm i \theta}+i T\right)\right|<(2 R+T)^{d_{\mathcal{L}}\left(\frac{1}{2}-a+2 R\right)} M(T) \exp \left(V^{*}(T)\right) .
$$

The same estimate holds also for $\overline{\mathcal{L}(\bar{z}+i T)}$. Thus

$$
\left|g\left(a+2 R e^{i \theta}\right)\right|<(2 R+T)^{d_{\mathcal{L}}\left(\frac{1}{2}-a+2 R\right)} M(T) \exp \left(V^{*}(T)\right) .
$$

We have estimated the term $\left|g\left(a+2 R e^{i \theta}\right)\right|$. Next we estimate the term $\left|\frac{1}{2 \pi \log 2} \int_{0}^{2 \pi} \log \right| g\left(a+2 R e^{i \theta}\right)|d \theta|$. Let

$$
\chi_{\log |g| \geq 0}=\left\{\begin{array}{l}
1, \text { if } \log |g| \geq 0 \\
0, \text { otherwise }
\end{array} .\right.
$$

Now, since $\int_{0}^{2 \pi} \log \left|g\left(a+2 R e^{i \theta}\right)\right| d \theta \geq 0$, we have

$$
\left|\int_{0}^{2 \pi} \log \right| g\left(a+2 R e^{i \theta}\right)|d \theta| \leq \int_{0}^{2 \pi} \chi_{\log |g| \geq 0} \log \left|g\left(a+2 R e^{i \theta}\right)\right| d \theta .
$$


Since for $\log \left|g\left(a+2 R e^{i \theta}\right)\right| \geq 0$ it holds that $\left|g\left(a+2 R e^{i \theta}\right)\right| \geq 1$, it is enough to know the upper bound of the term $\left|g\left(a+2 R e^{i \theta}\right)\right|$. Further, by the inequality (21) we have

$$
\begin{aligned}
& \int_{0}^{2 \pi} \chi_{\log |g| \geq 0} \log \left|g\left(a+2 R e^{i \theta}\right)\right| d \theta \\
& \quad<\int_{0}^{2 \pi}|\log |(2 R+T)^{d_{\mathcal{L}}\left(\frac{1}{2}-a+2 R\right)} M(T) \exp \left(V^{*}(T)\right)|| d \theta .
\end{aligned}
$$

The last step is to integrate the terms. By the definition of the functions $M(T)$ and $V^{*}(T)$ we have

$$
\begin{aligned}
|\log | & (2 R+T)^{d_{\mathcal{L}}\left(\frac{1}{2}-a+2 R\right)} M(T) \exp \left(V^{*}(T)\right)|| \\
\leq & d_{\mathcal{L}}\left(\frac{1}{2}-a+2 R\right) \log (2 T)+\log \frac{a_{1} \pi^{2}}{6}+\max \left\{\operatorname { m a x } \left\{\frac{5}{2} \log \left|\lambda Q^{2}\right|,\right.\right. \\
& \left.\left(\frac{1}{2}-a+2 R\right) \log \left|\lambda Q^{2}\right|\right\}-2 d_{\mathcal{L}}+\left|1-\frac{(a+2 R) i}{T-2 R}\right| d_{\mathcal{L}}\left(\frac{1}{2}-a+2 R\right) \\
& +d_{\mathcal{L}}(a+2 R)+\frac{a+2 R}{T-2 R}\left|\frac{\Im(\mu)}{2}\right|,\left(\frac{5}{2} d_{\mathcal{L}}+1\right) \log 2+k \log 3 \\
& \left.+\max \left\{0, \frac{5}{2} \log \left|\lambda Q^{2}\right|+\frac{5 \sqrt{5}}{2} d_{\mathcal{L}}+|\Im(\mu)|\right\}\right\}+V^{*}(T) .
\end{aligned}
$$

Thus we have

$$
\begin{aligned}
& \left|\frac{1}{2 \pi \log 2} \int_{0}^{2 \pi} \log \right| g\left(a+2 R e^{i \theta}\right)|d \theta| \\
& <\frac{1}{\log 2}\left(d_{\mathcal{L}}\left(\frac{1}{2}-a+2 R\right) \log (2 T)+\log \frac{a_{1} \pi^{2}}{6}+V^{*}(T)\right. \\
& +\max \left\{\max \left\{\frac{5}{2} \log \left|\lambda Q^{2}\right|,\left(\frac{1}{2}-a+2 R\right) \log \left|\lambda Q^{2}\right|\right\}-2 d_{\mathcal{L}}\right. \\
& +\left|1-\frac{(a+2 R) i}{T-2 R}\right| d_{\mathcal{L}}\left(\frac{1}{2}-a+2 R\right)+d_{\mathcal{L}}(a+2 R)+\frac{a+2 R}{T-2 R}\left|\frac{\Im(\mu)}{2}\right|, \\
& \left.\left.\left(\frac{5}{2} d_{\mathcal{L}}+1\right) \log 2+k \log 3+\max \left\{0, \frac{5}{2} \log \left|\lambda Q^{2}\right|+\frac{5 \sqrt{5}}{2} d_{\mathcal{L}}+|\Im(\mu)|\right\}\right\}\right) \\
& =R_{2}(T) \text {. }
\end{aligned}
$$

We define a new function $n(r)$ and estimate it. The estimate is used to estimate the integral $I_{3}(T, a, b)$. 
Theorem 4.5. Assume that $T$ satisfies the same conditions as in Theorem 4.2. Let $n(r)$ be the number of the zeros of the function $g(z)$ in $|z-a| \leq r$, where $g(z)$ is as in Lemma 4.4. Then $n(R)<R_{2}(T)+1$.

Proof. First we estimate the value of the function $n(R)$ with the function $g(z)$ and its integral. Then we estimate the previous terms and obtain $n(R)<R_{2}(T)+1$.

Since $\Im(z+i T)>0$ and $\Im(\bar{z}+i T)>0$ for $|z-a|<T$, the functions $\mathcal{L}(z+i T)$ and $\overline{\mathcal{L}(\bar{z}+i T)}$ are analytic in the disc $|z-a|<T$. Thus the function $g(z)$ is analytic in the disc $|z-a|<T$. We have

$$
\int_{0}^{2 R} \frac{n(r)}{r} d r \geq \int_{R}^{2 R} \frac{n(R)}{r} d r=n(R) \log (2) .
$$

By Jensen's formula

$$
\int_{0}^{2 R} \frac{n(r)}{r} d r=\frac{1}{2 \pi} \int_{0}^{2 \pi} \log \left|g\left(a+2 R e^{i \theta}\right)\right| d \theta-\log |g(a)| .
$$

Thus

$$
n(R) \leq \frac{1}{2 \pi \log 2} \int_{0}^{2 \pi} \log \left|g\left(a+2 R e^{i \theta}\right)\right| d \theta-\frac{\log |g(a)|}{\log 2} .
$$

From the previous formula and Lemma 4.4 we see that it is enough to estimate the terms

$$
\frac{1}{2 \pi \log 2} \int_{0}^{2 \pi} \log \left|g\left(a+2 R e^{i \theta}\right)\right| d \theta
$$

if $\int_{0}^{2 \pi} \log \left|g\left(a+2 R e^{i \theta}\right)\right| d \theta<0$ and $\frac{\log |g(a)|}{\log 2}$. By the assumptions for the number $a$ we have

$$
\Re(\mathcal{L}(a+i T))=\Re\left(1+\sum_{n=2}^{\infty} \frac{a(n)}{n^{a+i T}}\right) \in\left[\frac{1}{2}, \frac{3}{2}\right] .
$$

Since $g(a)=\Re(\mathcal{L}(a+i T))$, we have $|\log | g(a)|| \leq \log 2$. Thus

$$
\left|\frac{\log |g(a)|}{\log 2}\right| \leq 1
$$

Next we look at the case $\int_{0}^{2 \pi} \log \left|g\left(a+2 R e^{i \theta}\right)\right| d \theta<0$. By the definition of the function $n(R)$ we have $n(R) \geq 0$. Also by (22) and (23)

$$
-1 \leq-\left|\frac{\log |g(a)|}{\log 2}\right|+n(R) \leq \frac{1}{2 \pi \log 2} \int_{0}^{2 \pi} \log \left|g\left(a+2 R e^{i \theta}\right)\right| d \theta .
$$

Thus

$$
\left|\frac{1}{2 \pi \log 2} \int_{0}^{2 \pi} \log \right| g\left(a+2 R e^{i \theta}\right)|d \theta| \leq 1
$$


if $\int_{0}^{2 \pi} \log \left|g\left(a+2 R e^{i \theta}\right)\right| d \theta<0$. By (22), (23), (24) and Lemma 4.4 we get the result

$$
n(R)<R_{2}(T)+1 .
$$

In the following theorem we estimate the integral $I_{3}(T, a, b)=\int_{b}^{a} \mathcal{L}(\sigma+$ $i T) d \sigma$ using the previous theorem.

Theorem 4.6. If $T$ satisfies the same conditions as in Theorem 4.2, then

$$
\left|I_{3}(T, a, b)\right|<\pi R\left(R_{2}(T)+2\right) .
$$

Proof. Assume that the function $\Re(\mathcal{L}(\sigma+i T))$ has $N$ zeros for $b \leq \sigma \leq a$. Now the sign of the $\Re(\mathcal{L}(\sigma+i T))$ changes at most $N+1$ times in the interval $\sigma \in[b, a]$. We can divide the interval $[b, a]$ to $N+1$ parts where $\Re(\mathcal{L}(\sigma+i T))$ is of constant sign. When we sum the maximum absolute values of the argument in each of these intervals, we get

$$
|\arg (\mathcal{L}(\sigma+i T))| \leq \pi(N+1) .
$$

Let $n(R)$ and $g(z)$ be as in Theorem 4.5. Since $(b, a) \subseteq\{z:|z-a| \leq R\}$ and $g(\sigma)=\Re(\mathcal{L}(\sigma+i T))$, we have $N \leq n(R)$. Thus

$$
\pi(N+1) \leq \pi(n(R)+1) .
$$

By Theorem 4.5 we have $\pi(n(R)+1)<\pi\left(R_{2}(T)+2\right)$. It follows that

$$
\left|I_{3}(T, a, b)\right| \leq \int_{b}^{a}|\arg \mathcal{L}(\sigma+i T)| d \sigma<\pi R\left(R_{2}(T)+2\right) .
$$

Remark 4.7. Since $(b+1, a) \subset(b, a)$ it also holds that $\left|I_{3}(T, a, b+1)\right|<$ $\pi R\left(R_{2}(T)+2\right)$.

\section{Main result}

In this section we prove the explicit version of the Riemann-von Mangoldt type formula for the functions of the set $S$. Let $\mathcal{N}_{\mathcal{L}}^{+}\left(T_{0}, T\right)$ and $\mathcal{N}_{\mathcal{L}}^{-}\left(T_{0}, T\right)$ be the number of the non-trivial zeros $\rho$ of the function $\mathcal{L}(s)$ with $T_{0}<\Im(\rho) \leq$ $T$ and $-T \leq \Im(\rho)<-T_{0}$ respectively. First we combine the results from the Sections 2, 3 and 4 to estimate the functions $\mathcal{N}_{\mathcal{L}}^{+}\left(T_{0}, T\right)$ and $\mathcal{N}_{\mathcal{L}}^{-}\left(T_{0}, T\right)$. We remember that $a_{1}$ is a constant such that for all $n$ we have $|a(n)| \leq a_{1} n$. Also, $a$ is a real number for which $a>2$ and

$$
\sum_{n=2}^{\infty} \frac{a_{1}}{n^{a}}<\frac{1}{2}
$$


and $b<-3$ is a negative real number which has the following property:

$$
\sum_{n=2}^{\infty} \frac{a_{1}}{n^{-b-1}}<1
$$

We have defined that $R=a-b$. The constants $d_{\mathcal{L}}, \lambda, Q, f, \mu_{j}$ and $\lambda_{j}$ depend on the function $\mathcal{L}$ and are defined at the beginning of the Section 1. The function $R_{1}\left(T_{0}, T, b\right)$ is defined in the formula (8) of the Section 3.2 and the function $R_{2}(t)$ is defined in the formula (20) of the Section 4.2, Let

$$
\begin{aligned}
R_{\mathcal{L}}\left(T_{0}, T\right) & \\
= & \frac{d_{\mathcal{L}}}{2 \pi} T_{0} \log \frac{T_{0}}{e}+\frac{T_{0}}{2 \pi}\left|\log \left(\lambda Q^{2}\right)\right|+\frac{R_{1}\left(T_{0}, T, b\right)}{2 \pi}+\frac{\pi}{3 \log 2} \\
& +\left(R-\frac{1}{2}\right)\left(R_{2}\left(T_{0}\right)+R_{2}(T)+4\right)+f \cdot\left(\left|(b+1) \max _{j}\left\{\lambda_{j}\right\}+\min _{j}\left\{\Re\left(\mu_{j}\right)\right\}\right|\right. \\
& \left.-b \max _{j}\left\{\lambda_{j}\right\}+(b+1) \min _{j}\left\{\lambda_{j}\right\}-\min _{j}\left\{\Re\left(\mu_{j}\right)\right\}+\max _{j}\left\{\Re\left(\mu_{j}\right)\right\}\right) .
\end{aligned}
$$

First we prove a useful lemma and then we use it to prove the main result.

Lemma 5.1. Assume that $T_{0}$ satisfies the same conditions as $T$ in Theorem 4.2 and $T>T_{0}$ is a real number. Then

$$
\left|\mathcal{N}_{\mathcal{L}}^{ \pm}\left(T_{0}, T\right)-\frac{d_{\mathcal{L}}}{2 \pi} T \log \frac{T}{e}-\frac{T}{2 \pi} \log \left(\lambda Q^{2}\right)\right|<R_{\mathcal{L}}\left(T_{0}, T\right) .
$$

Proof. Since the functions $\mathcal{L}(\bar{s})$ and $\overline{\mathcal{L}(\bar{s})}=\sum_{n=1}^{\infty} \frac{\overline{a(n)}}{n^{s}}$ have the same zeros, we need to prove the claim only for the function $\mathcal{N}_{\mathcal{L}}^{+}\left(T_{0}, T\right)$. By Lemma ?? for the zeros $\rho$ of the function $\mathcal{L}$ we have

$$
2 \pi \sum_{\substack{T_{0}<\Im(\rho) \leq T \\ \Re(\rho)>b}}(\Re(\rho)-b)=I_{1}\left(T_{0}, T, b\right)-I_{2}\left(T_{0}, T, a\right)-I_{3}\left(T_{0}, a, b\right)+I_{3}(T, a, b) .
$$

We can subtract the formula containing $b+1$ from the formula containing $b$ and get

$$
\begin{aligned}
& 2 \pi \mathcal{N}_{\mathcal{L}}^{+}\left(T_{0}, T\right)+2 \pi \sum_{\substack{T_{0}<\Im(\rho) \leq T \\
0>\Re(\rho)>b+1}} 1+2 \pi \sum_{\substack{T_{0}<\Im(\rho) \leq T \\
b+1 \geq \Re(\rho)>b}}(\Re(\rho)-b) \\
& =I_{1}\left(T_{0}, T, b\right)-I_{1}\left(T_{0}, T, b+1\right)-I_{3}\left(T_{0}, a, b\right) \\
& \quad+I_{3}\left(T_{0}, a, b+1\right)+I_{3}(T, a, b)-I_{3}(T, a, b+1) .
\end{aligned}
$$


By Theorems 3.5, 4.6 and Remark 4.7 we have

$$
\begin{aligned}
& \left|\mathcal{N}_{\mathcal{L}}^{+}\left(T_{0}, T\right)-\frac{d_{\mathcal{L}}}{2 \pi} T \log \frac{T}{e}-\frac{T}{2 \pi} \log \left(\lambda Q^{2}\right)\right| \\
& \quad<\frac{d_{\mathcal{L}}}{2 \pi} T_{0} \log \frac{T_{0}}{e}+\frac{T_{0}}{2 \pi}\left|\log \left(\lambda Q^{2}\right)\right|+\frac{R_{1}\left(T_{0}, T, b\right)}{2 \pi}+\frac{\pi}{3 \log 2} \\
& \quad+\left(R-\frac{1}{2}\right)\left(R_{2}\left(T_{0}\right)+R_{2}(T)+4\right)+\left|\sum_{\substack{T_{0}<\Im(\rho) \leq T \\
0>\Re(\rho)>b+1}} 1+\sum_{\substack{T_{0}<\Im(\rho) \leq T \\
b-1 \geq \Re(\rho)>b}}(\Re(\rho)-b)\right| .
\end{aligned}
$$

Since all the trivial zeros are of the form $s=-\frac{l+\mu_{j}}{\lambda_{j}}$, where $l=0,1,2, \ldots$ and $j \in[1, f]$, we have

$$
\begin{aligned}
& \left|\sum_{\substack{T_{0}<\Im(\rho) \leq T \\
0>\Re(\rho)>b+1}} 1+\sum_{\substack{T_{0}<\Im(\rho) \leq T \\
b+1 \geq \Re(\rho)>b}}(\Re(\rho)-b)\right| \\
& <f \cdot\left(\left|(b+1) \max _{j}\left\{\lambda_{j}\right\}+\min _{j}\left\{\Re\left(\mu_{j}\right)\right\}\right|\right. \\
& \left.\quad-b \max _{j}\left\{\lambda_{j}\right\}+(b+1) \min _{j}\left\{\lambda_{j}\right\}-\min _{j}\left\{\Re\left(\mu_{j}\right)\right\}+\max _{j}\left\{\Re\left(\mu_{j}\right)\right\}\right) .
\end{aligned}
$$

The claim follows from the inequalities (25) and (26).

Next we prove the main result by estimating the term $R_{\mathcal{L}}\left(T_{0}, T\right)$. We want that it holds that

$$
\left|R_{\mathcal{L}}\left(T_{0}, T\right)\right| \leq c_{\mathcal{L}, 1} \log T+c_{\mathcal{L}, 2}\left(T_{0}\right)+\frac{c_{\mathcal{L}, 3}\left(T_{0}\right)}{T},
$$

where the terms $c_{\mathcal{L}, j}\left(T_{0}\right)$ are real numbers which depend on the function $\mathcal{L}$ and the number $T_{0}$ and the real number $c_{\mathcal{L}, 1}$ depends only on the function $\mathcal{L}$. To shorten our notation we define that

$$
\begin{aligned}
h_{\mathcal{L}, 1}= & (1-\alpha)\left(\max \left\{\frac{5}{2} \log \left|\lambda Q^{2}\right|,\left(\frac{1}{2}-a+2 R\right) \log \left|\lambda Q^{2}\right|\right\}+d_{\mathcal{L}}\left(-\frac{3}{2}+4 R\right)\right) \\
& +\alpha\left(\left(\frac{5}{2} d_{\mathcal{L}}+1\right) \log 2+k \log 3+\max \left\{0, \frac{5}{2} \log \left|\lambda Q^{2}\right|+\frac{5 \sqrt{5}}{2} d_{\mathcal{L}}+|\Im(\mu)|\right\}\right)
\end{aligned}
$$

and

$$
h_{\mathcal{L}, 2}=(1-\alpha)\left(d_{\mathcal{L}}\left(\frac{1}{2}-a+2 R\right)(a+2 R)+(a+2 R)\left|\frac{\Im(\mu)}{2}\right|\right)
$$

where the number $\alpha \in\{0,1\}$. If the sum $h_{\mathcal{L}, 1}+\frac{h_{\mathcal{L}, 2}}{T_{0}-2 R}$ is bigger for $\alpha=0$ than $\alpha=1$ then $\alpha=0$. Otherwise $\alpha=1$. Using this notation we obtain the main result: 
Theorem 5.2. Suppose that $T_{0}$ and $T$ satisfy the same conditions as in Lemma 5.1, Then we have

$$
\left|R_{\mathcal{L}}\left(T_{0}, T\right)\right| \leq c_{\mathcal{L}, 1}\left(T_{0}\right) \log T+c_{\mathcal{L}, 2}\left(T_{0}\right)+\frac{c_{\mathcal{L}, 3}\left(T_{0}\right)}{T},
$$

where

$$
\begin{aligned}
c_{\mathcal{L}, 1}= & \frac{1}{2 \pi}\left(-7 \frac{d_{\mathcal{L}}}{2}(2 b+1)+2\left|-d_{\mathcal{L}} b+\frac{\Im(\mu) i}{2}\right|+2 d_{\mathcal{L}}+S(1, e, b)\right) \\
& +\frac{1}{\log 2}\left(R-\frac{1}{2}\right) d_{\mathcal{L}}\left(\frac{1}{2}-a+2 R\right), \\
c_{\mathcal{L}, 2}\left(T_{0}\right)= & \frac{d_{\mathcal{L}}}{2 \pi} T_{0} \log \frac{T_{0}}{e}+\frac{T_{0}}{2 \pi}\left|\log \left(\lambda Q^{2}\right)\right|+\frac{\pi}{3 \log 2}+4 R-2 \\
& +\frac{3 d_{\mathcal{L}}\left(b^{2}+b\right)}{2 \pi T_{0}}+f \cdot\left(\left|(b+1) \max _{j}\left\{\lambda_{j}\right\}+\min _{j}\left\{\Re\left(\mu_{j}\right)\right\}\right|\right. \\
& \left.-b \max _{j}\left\{\lambda_{j}\right\}+(b+1) \min _{j}\left\{\lambda_{j}\right\}-\min _{j}\left\{\Re\left(\mu_{j}\right)\right\}+\max _{j}\left\{\Re\left(\mu_{j}\right)\right\}\right) \\
& +\frac{1}{2 \pi} R_{1}\left(T_{0}, 1, b\right)+\left(R-\frac{1}{2}\right)\left(R_{2}\left(T_{0}\right)+d_{\mathcal{L}}\left(\frac{1}{2}-a+2 R\right)\right) \\
& +\frac{1}{\log 2}\left(R-\frac{1}{2}\right)\left(\log \frac{a_{1} \pi^{2}}{6}+h_{\mathcal{L}, 1}\right)
\end{aligned}
$$

and

$$
c_{\mathcal{L}, 3}\left(T_{0}\right)=\frac{1}{\log 2}\left(R-\frac{1}{2}\right) \frac{T_{0}}{T_{0}-2 R}\left(V^{*}(2 R+1)+h_{\mathcal{L}, 2}\right) .
$$

Proof. Since $\left|1-\frac{(a+2 R) i}{T_{0}-2 R} \leq 1+\frac{a+2 R}{T_{0}-2 R}\right|$ and $\frac{1}{T-2 R} \leq \frac{T_{0}}{\left(T_{0}-2 R\right) T}$, the claim follows from the definition of the term $R_{\mathcal{L}}\left(T_{0}, T\right)$.

Using the main result we can prove a useful corollary. If we know the number of up to height $T_{0}$, we can also estimate the the number of zeros up to height $T$. Let $\mathcal{N}_{\mathcal{L}}^{+}(t)$ and $\mathcal{N}_{\mathcal{L}}^{-}(t)$ denote the number of the non-trivial zeros of the function $\mathcal{L}$ for which $0 \leq \Im(\rho) \leq t$ and $-t \leq \Im(\rho) \leq 0$ respectively. We also notice that by [18] we have that $\mathcal{N}_{\mathcal{L}}^{ \pm}(T) \sim \frac{d_{\mathcal{L}}}{2 \pi} T \log T$ and thus the numbers $\mathcal{N}_{\mathcal{L}}^{ \pm}\left(T_{0}\right)$ are finite. Using these properties we obtain the following corollary:

Corollary 5.3. Suppose that $T_{0}$ and $T$ satisfy the same conditions as in Lemma 5.1. Since for all positive real numbers $c$ it holds that $c \leq c \log T$, by Theorem 5.2 we get

$$
\left|R_{\mathcal{L}}\left(T_{0}, T\right)+\mathcal{N}_{\mathcal{L}}^{ \pm}\left(T_{0}\right)\right| \leq c_{\mathcal{L}, 1} \log T+C_{\mathcal{L}, 2}\left(T_{0}\right)+\frac{c_{\mathcal{L}, 3}\left(T_{0}\right)}{T},
$$

for example, when $C_{\mathcal{L}, 2}\left(T_{0}\right)=c_{\mathcal{L}, 2}\left(T_{0}\right)+\max \left\{\mathcal{N}_{\mathcal{L}}^{+}\left(T_{0}\right), \mathcal{N}_{\mathcal{L}}^{-}\left(T_{0}\right)\right\}$. 
Furthermore, we also would like to note one interesting and useful result. For the number of zeros in the interval $(T, 2 T]$ we obtain a formula with the error term where coefficients of the terms $\log T, 1$ and $\frac{1}{T}$ don't depend on the number $T$.

Remark 5.4. Using similar methods as in the proof of Lemma 5.1 and Theorem 5.2 we can prove that

$$
\left|\mathcal{N}_{\mathcal{L}}^{ \pm}(T, 2 T)-\frac{d_{\mathcal{L}}}{2 \pi} T \log \frac{4 T}{e}-\frac{T}{2 \pi} \log \left(\lambda Q^{2}\right)\right| \leq c_{1} \log T+c_{2}+\frac{c_{3}}{T},
$$

where

$$
\begin{gathered}
c_{1}=\frac{d_{\mathcal{L}}}{\log 2}(2 R-1)\left(\frac{1}{2}-a+2 R\right) \\
c_{2}=\frac{\log 2}{2 \pi}\left(-\frac{7 d_{\mathcal{L}}}{2}(2 b+1)+2\left|-d_{\mathcal{L}} b+\frac{\Im(\mu) i}{2}\right|+2 d_{\mathcal{L}}\right)+\frac{S(1,2, b)}{2 \pi}+\frac{2 \pi}{3 \log 2} \\
+4 R-2+(2 R-1)\left(\frac{3 d_{\mathcal{L}}}{2}\left(\frac{1}{2}-a+2 R\right)+\frac{1}{\log 2}\left(\log \frac{a_{1} \pi^{2}}{6}++h_{\mathcal{L}, 1}\right)\right)
\end{gathered}
$$

and

$$
c_{3}=\frac{3 d_{\mathcal{L}}\left(b^{2}+b\right)}{4 \pi}+\frac{3 T_{0}}{2\left(T_{0}-2 R\right) \log 2}\left(R-\frac{1}{2}\right)\left(V^{*}(2 R+1)+h_{\mathcal{L}, 2}\right) .
$$

\section{Example: $L$-function associated with a holomor- phic newform}

In this section we give examples of the values of the terms $c_{\mathcal{L}, 1}, c_{\mathcal{L}, j}\left(T_{0}\right)$ and $c_{j}$ which are defined in Section 5. Since we have estimated these terms for a general set which contains $L$-functions other than the Riemann zeta function and Dirichlet $L$-functions, the estimates of the term $c_{\mathcal{L}, 1}$ and $c_{\mathcal{L}, j}\left(T_{0}\right)$ for these functions are not as strong as previous estimates, see [1] and [21.

Let $g$ be a newform of even weight $\kappa$ for some congruence subgroup

$$
\Gamma_{0}(N)=\left\{\left(\begin{array}{ll}
A & B \\
C & D
\end{array}\right) \in S L_{2}(\mathbb{Z}): C \equiv 0 \quad \bmod N\right\}
$$

where $N$ is a positive integer and $S L_{2}(\mathbb{Z})$ is a set of $2 \times 2$ matrices with integer entries and which determinant is 1 . We also assume that for $z \in \mathbb{H}$ the function $g$ has a Fourier expansion

$$
g(z)=\sum_{n=1}^{\infty} c(n) \exp (2 \pi i n z) .
$$

We define

$$
\mathcal{L}(s)=\sum_{n=1}^{\infty} \frac{a(n)}{n^{s}},
$$


where $a(n)=c(n) n^{\frac{1-\kappa}{2}}$. The function $\mathcal{L}(s)$ satisfies the equation

$$
\Lambda_{\mathcal{L}}(s)=\mathcal{L}(s)\left(\frac{\sqrt{N}}{2 \pi}\right)^{s} \Gamma\left(s+\frac{\kappa-1}{2}\right),
$$

where

$$
\Lambda_{\mathcal{L}}(s)=i^{\kappa} \Lambda_{\mathcal{L}}(1-s)
$$

Hence, we can choose

$$
f=1, Q=\frac{\sqrt{N}}{2 \pi}, \lambda_{j}=1, \omega=i^{\kappa} \text { and } \mu_{j}=\frac{\kappa-1}{2} .
$$

Thus we also have $d_{\mathcal{L}}=2, \lambda=1$ and $\mu=4-2 \kappa$. We also have $k=0$. By Deligne [6, 7] $|a(n)| \leq 1$ and we can choose that $a_{1}=1, a=3$ and $b=-4$. By Theorem 5.2 we have $T_{0} \geq 15+\kappa$ and

$$
\begin{aligned}
& c_{\mathcal{L}, 1}=\frac{299}{2 \log 2}+\frac{1}{2 \pi}\left(3 \kappa^{2}-2 \kappa+\frac{217}{3}\right. \\
&\left.+\frac{1}{12}\left(\sec ^{2}\left(\frac{\arg (4+(\kappa+1) i)}{2}\right)+\sec ^{2}\left(\frac{\arg (3+(\kappa+1) i)}{2}\right)\right)\right), \\
& c_{\mathcal{L}, 2(15+\kappa)=} \frac{1}{\pi}(15+\kappa) \log \frac{15+\kappa}{e}+\frac{15+\kappa}{2 \pi}\left|\log \frac{N}{4 \pi^{2}}\right|+\frac{\pi}{3 \log 2}+\frac{353}{2} \\
&+ \frac{36}{\pi(15+\kappa)}+\left|\frac{\kappa-7}{2}\right|+\frac{72}{2 \pi(15+\kappa)} \\
&-\frac{\log (15+\kappa)}{2 \pi}\left(\frac{9 \kappa^{2}-6 \kappa+217}{3}+\right. \\
&\left.+\frac{1}{12}\left(\sec ^{2}\left(\frac{\arg (4+(\kappa+1) i)}{2}\right)+\sec ^{2}\left(\frac{\arg (3+(\kappa+1) i)}{2}\right)\right)\right) \\
&+\frac{13}{12(1+\kappa) \log 2}\left(9 \kappa^{2}-6 \kappa+10+\frac{1}{2} \sec ^{2}\left(\frac{\arg (-17+(\kappa+1) i)}{2}\right)\right) \\
&+\frac{299}{2 \log 2}\left(\log (30+2 \kappa)+\left|1-\frac{17 i}{\kappa+1}\right|\right) \\
&+\frac{13}{2 \log 2}\left(2 \log \frac{\pi^{2}}{6}+2 \max \left\{\frac{5}{2} \log \frac{N}{4 \pi^{2}}, \frac{23}{2} \log \frac{N}{4 \pi^{2}}\right\}+83\right)
\end{aligned}
$$

and

$$
\begin{aligned}
c_{\mathcal{L}, 3}(15+\kappa)= & \frac{13}{12 \log 2} \frac{15+\kappa}{1+\kappa}\left(9 \kappa^{2}-6 \kappa+2356\right. \\
& \left.+\frac{1}{2} \sec ^{2}\left(\frac{\arg (-17+(\kappa+1) i)}{2}\right)\right) .
\end{aligned}
$$


Furthermore, by Remark 5.4 we also have

$$
\begin{gathered}
c_{1}=\frac{299}{\log 2}, \\
c_{2}=\frac{2 \pi}{3 \log 2}+923+\frac{\log 2}{6 \pi}\left(9 \kappa^{2}-6 \kappa+217\right. \\
\left.+\frac{1}{4}\left(\sec ^{2}\left(\frac{\arg (4+(\kappa+1) i)}{2}\right)+\sec ^{2}\left(\frac{\arg (3+(\kappa+1) i)}{2}\right)\right)\right) \\
+\frac{13}{\log 2}\left(\log \frac{\pi^{2}}{6}+\max \left\{\frac{5}{2} \log \frac{N}{4 \pi^{2}}, \frac{23}{2} \log \frac{N}{4 \pi^{2}}\right\}+53\right)
\end{gathered}
$$

and

$$
\begin{aligned}
c_{3}= & \frac{18}{\pi}+\frac{13(15+\kappa)(17+3 \kappa)}{4(1+\kappa)(8+\kappa) \log 2}\left(\frac { 1 } { 6 ( 1 + \kappa ) } \left(9 \kappa^{2}-6 \kappa+10\right.\right. \\
& \left.\left.+\frac{1}{2} \sec ^{2}\left(\frac{\arg (-17+(\kappa+1) i)}{2}\right)\right)+391\right) .
\end{aligned}
$$

We can see different values of the ceiling function of the numbers $c_{\mathcal{L}, 1}$, $c_{\mathcal{L}, j}\left(T_{0}\right)$ and $c_{j}$ from Table 1 . 
Table 1: Different values of the terms $c_{\mathcal{L}, 1}, c_{\mathcal{L}, j}\left(T_{0}\right)$ and $c_{j}$

\begin{tabular}{rrrrrrrrr}
\hline$N$ & $\kappa$ & $T_{0}$ & $c_{\mathcal{L}, 1}$ & $c_{\mathcal{L}, 2}\left(T_{0}\right)$ & $c_{\mathcal{L}, 3}\left(T_{0}\right)$ & $c_{1}$ & $c_{2}$ & $c_{3}$ \\
\hline 1 & 12 & 27 & 293 & 1945 & 11637 & 432 & 1811 & 10506 \\
1 & 34 & 49 & 769 & 415 & 27478 & 432 & 2141 & 8183 \\
1 & 36 & 51 & 835 & 172 & 29742 & 432 & 2187 & 8133 \\
1 & 38 & 53 & 905 & -91 & 32127 & 432 & 2235 & 8092 \\
1 & 40 & 55 & 979 & -374 & 34631 & 432 & 2286 & 8060 \\
1 & 50 & 65 & 1405 & -2087 & 48918 & 432 & 2582 & 7983 \\
2 & 8 & 23 & 256 & 2112 & 11554 & 432 & 1817 & 12323 \\
2 & 10 & 25 & 272 & 2040 & 11375 & 432 & 1829 & 11247 \\
11 & 2 & 17 & 229 & 2941 & 21661 & 432 & 1879 & 24239 \\
11 & 10 & 25 & 272 & 2113 & 11375 & 432 & 1909 & 11247 \\
11 & 12 & 27 & 293 & 2047 & 11637 & 432 & 1923 & 10506 \\
11 & 36 & 51 & 835 & 265 & 29742 & 432 & 2299 & 8133 \\
11 & 38 & 53 & 905 & 1 & 32127 & 432 & 2347 & 8092 \\
11 & 40 & 55 & 979 & -282 & 34631 & 432 & 2399 & 8060 \\
21 & 6 & 21 & 243 & 2314 & 12460 & 432 & 1919 & 14017 \\
21 & 8 & 23 & 256 & 2214 & 11554 & 432 & 1928 & 12323 \\
40 & 2 & 17 & 229 & 3000 & 21661 & 432 & 1942 & 24239 \\
40 & 6 & 21 & 243 & 2345 & 12460 & 432 & 1951 & 14017 \\
40 & 36 & 51 & 835 & 317 & 29742 & 432 & 2362 & 8133 \\
40 & 38 & 53 & 905 & 53 & 32127 & 432 & 2410 & 8092 \\
63 & 36 & 51 & 835 & 419 & 29742 & 432 & 2460 & 8133 \\
63 & 38 & 53 & 905 & 155 & 32127 & 432 & 2508 & 8092 \\
64 & 36 & 51 & 835 & 422 & 29742 & 432 & 2463 & 8133 \\
64 & 38 & 53 & 905 & 159 & 32127 & 432 & 2512 & 8092 \\
64 & 40 & 55 & 979 & -125 & 34631 & 432 & 2563 & 8060 \\
\hline & & & & & & & &
\end{tabular}




\section{References}

[1] R. J. Backlund. Über die Nullstellen der Riemannschen Zetafunction. Acta Mathematica, 41:345-375, 1916.

[2] E. Carneiro and V. Chandee. Bounding $S(t)$ and $S_{1}(t)$ on the Riemann hypothesis. Math. Ann., 356:939-968, 2013.

[3] E. Carneiro, V. Chandee, and M. B. Milinovich. A note on the zeros of zeta and L-functions. Math. Z., 281:315-332, 2015.

[4] E. Carneiro and R. H. Finder. On the argument of $L$-functions. Bull. Braz. Math. Soc., 46:601-620, 2015.

[5] J. B. Conrey and A. Ghosh. On the Selberg class of Dirichlet series: small degrees. Duke Math. J., 72:673-693, 1993.

[6] P. Deligne. La Conjecture de Weil. I. Publ. I.H.E.S., 43:273-307, 1974.

[7] P. Deligne. La Conjecture de Weil. II. Publ. I.H.E.S., 52:137-252, 1980.

[8] R. H. Finder. Variation-diminishing maximal operators and the argument of L-functions. PhD thesis, Instituto de Matemática Pura e Aplicada, 2016.

[9] G. França and A. LeClair. Transcendental equations satisfied by the individual zeros of Riemann $\zeta$, Dirichlet and modular $L$-functions. Commun. Number Theory Phys., 9:1-50, 2015.

[10] A. Fujii. On the zeros of Dirichlet L-functions I. Transactions of the American Math. Soc., 196:225-235, 1974.

[11] H. Iwaniec and E. Kowalski. Analytic Number Theory, volume 53 of American Math. Soc, Colloq. Publ. American Math. Soc., 2004.

[12] K. S. McCurley. Explicit estimates for the error term in the prime number theorem for arithmetic progressions. Math. Comp., 42:265285,1984 .

[13] A. Selberg. Old and new conjectures and results about a class of Dirichlet series. In E. Bombieri et al., editors, Proc. Amalfi Conf. Analytic Number Theory, pages 367-385. Universitia di Salerno, 1992.

[14] A. Selberg. Contributions to the theory of Dirichlet's $L$-fuctions. $S k r$. Norske Vid. Akad. Oslo. I, (3):2-62, 1946.

[15] R. E. Shafer, L. S. Grinstein, D. C. B. Marsh, and J. D. E. Konhauser. Problems and solutions: Solutions of elementary problems: E1867. The American Mathematical Monthly, 74(6):726-727, 1967. 
[16] L. Smajlović. On Li's criterion for the Riemann hypothesis for the Selberg class. J. Number Theory, 130:828-851, 2010.

[17] J. Steuding. On the value-distribution of $L$-functions. Fiz. Mat. Fak. Moksl. Semin. Darb., 6:87-119, 2003.

[18] J. Steuding. Value-Distribution of L-Functions. Lecture Notes in Mathematics. Springer-Verlag Berlin Heidelberg, 1 edition, 2007.

[19] T. J. Stieltjes. Sur le developpment de $\log \Gamma(a)$. Journal de Mathematiques, 5:425-444, 1889.

[20] T. S. Trudgian. An improved upper bound for the argument of the Riemann zeta-function on the critical line II. J. Number Theory, 134:280$292,2014$.

[21] T. S. Trudgian. An improved upper bound for the error in the zero-counting formulae for Dirichlet $L$-functions and Dedekind zetafunctions. Math. Comp., 84:1439-1450, 2015.

[22] H. von Mangoldt. Über die Anzahl der Primzahlen unter einer gegebenen Grösse. J. reine angew. Math., 114:255-305, 1895.

[23] H. von Mangoldt. Zur Verteilung der Nullstellen der Riemannschen Funktion $\zeta(t)$. Math. Ann., 60:1-19, 1905. 